\title{
Almost Product Evaluation of Hankel Determinants
}

\author{
Ömer Eğecioğlu \\ Department of Computer Science \\ University of California, Santa Barbara CA 93106 \\ omer@cs.ucsb.edu \\ Timothy Redmond \\ Stanford Medical Informatics, Stanford University, \\ Stanford, CA 94305 \\ tredmond@stanford.edu \\ Charles Ryavec \\ College of Creative Studies, \\ University of California, Santa Barbara CA 93106 \\ ryavec@math . ucsb . edu
}

Submitted: Apr 25, 2007; Accepted: Dec 18, 2007; Published: Jan 1, 2008

Mathematics Subject Classifications: 05A10, 05A15, 05A19, 05E35, 11C20, 11B65

\begin{abstract}
An extensive literature exists describing various techniques for the evaluation of Hankel determinants. The prevailing methods such as Dodgson condensation, continued fraction expansion, LU decomposition, all produce product formulas when they are applicable. We mention the classic case of the Hankel determinants with binomial entries $\left(\begin{array}{c}3 k+2 \\ k\end{array}\right)$ and those with entries $\left(\begin{array}{c}3 k \\ k\end{array}\right)$; both of these classes of Hankel determinants have product form evaluations. The intermediate case, $\left(\begin{array}{c}3 k+1 \\ k\end{array}\right)$ has not been evaluated. There is a good reason for this: these latter determinants do not have product form evaluations. In this paper we evaluate the Hankel determinant of $\left(\begin{array}{c}3 k+1 \\ k\end{array}\right)$. The evaluation is a sum of a small number of products, an almost product. The method actually provides more, and as applications, we present the salient points for the evaluation of a number of other Hankel determinants with polynomial entries, along with product and almost product form evaluations at special points.
\end{abstract}

\section{Introduction}

A determinant

$$
H_{n}=\operatorname{det}\left[a_{i, j}\right]_{0 \leq i, j \leq n}
$$


whose entries satisfy

$$
a_{i, j}=a_{i+j}
$$

for some sequence $\left\{a_{k}\right\}_{k \geq 0}$ is said to be a Hankel determinant. Thus $H_{n}$ is the determinant of a special type of $(n+1) \times(n+1)$ symmetric matrix.

In various cases of Hankel determinant evaluations, special techniques such as Dodgson condensation, continued fraction expansion, and LU decomposition are applicable. These methods provide product formulas for a large class of Hankel determinants. A modern treatment of the theory of determinant evaluation including Hankel determinants as well as a substantial bibliography can be found in Krattenthaler [7, 8].

The product form determinants of special note are those whose factors have some particular attraction. Factorials and other familiar combinatorial entities that appear as factors have an especially pleasing quality, and we find an extensive literature devoted to the evaluation of classes of Hankel determinants as such products.

Several classical Hankel determinants involve entries that are binomial coefficients or expressions closely related to binomial coefficients. Perhaps the most well-known of these is where $a_{k}=\left(\begin{array}{c}2 k+1 \\ k\end{array}\right)$, and $a_{k}=\frac{1}{2 k+1}\left(\begin{array}{c}2 k+1 \\ k\end{array}\right)$, for which $H_{n}=1$ for all $n$. The binomial entries

$$
a_{k}=\left(\begin{array}{c}
3 k \\
k
\end{array}\right), \quad a_{k}=\left(\begin{array}{c}
3 k+2 \\
k
\end{array}\right)
$$

also yield product evaluations for the corresponding $H_{n}$ as we give in (4) and (3). In fact, product formulas have been shown to exist for a host of other cases (see Gessel and Xin $[4]$ ), and we only mention

$$
a_{k}=\frac{1}{3 k+1}\left(\begin{array}{c}
3 k+1 \\
k
\end{array}\right), \quad a_{k}=\frac{9 k+14}{(3 k+4)(3 k+5)}\left(\begin{array}{c}
3 k+2 \\
k+1
\end{array}\right)
$$

as representatives.

However, within the restricted class of Hankel determinants defined by the binomial coefficients

$$
a_{k}=a_{k}^{(\beta, \alpha)}=\left(\begin{array}{c}
\beta k+\alpha \\
k
\end{array}\right),
$$

parametrized by a pair of integers $\beta>0$ and $\alpha$, it is a rare phenomenon that the determinant evaluations are in product form. An extensive check of Hankel determinants of sequences $a_{k}$ in the form (2) suggests that there is no product formula for $H_{n}$ in general for such binomial sequences. In fact, it would seem that the instances for which $H_{n}$ has a product form can be enumerated in full:

(i) $\beta=1, \alpha$ arbitrary,

(ii) $\beta=2, \alpha=0,1,2,3,4$,

(iii) $\beta=3, \alpha=0,2$. 
All the other cases are likely not products, but in any event, the question remains open: are evaluations possible in these cases?

Let $H_{n}^{(\beta, \alpha)}$ denote the $(n+1) \times(n+1)$ Hankel determinant with entries $a_{k}^{(\beta, \alpha)}$ as defined in (2). We will also use the term $(\beta, \alpha)$-case to refer to the evaluation of $H_{n}^{(\beta, \alpha)}$.

Numerical data indicates the intriguing possibility that the $H_{n}^{(\beta, \alpha)}$ might be evaluated as a sum of a small number of products, where "small" would mean $O\left(n^{d}\right)$ summands for some fixed $d=d(\beta, \alpha)$. We refer to such an evaluation as an almost product.

The evidence of an almost product evaluation of $H_{n}^{(\beta, \alpha)}$ is most pronounced for $\beta=3$, and we begin with some sample data.

For the $(3,2)$-case the Hankel determinants evaluate to

$$
\begin{aligned}
H_{10}^{(3,2)=} & 2^{2} \cdot 3 \cdot 7^{3} \cdot 37 \cdot 41^{2} \cdot 43^{3} \cdot 47^{3} \cdot 53^{2} \cdot 59 \cdot 61 \\
H_{20}^{(3,2)=} & 3^{7} \cdot 11 \cdot 17 \cdot 29^{2} \cdot 31 \cdot 67 \cdot 71^{2} \cdot 73^{3} \cdot 79^{5} \cdot 83^{6} \cdot 89^{6} \cdot 97^{5} \cdot 101^{4} \cdot 103^{4} . \\
& 107^{3} \cdot 109^{3} \cdot 113^{2} \\
H_{30}^{(3,2)=} & 2^{10} \cdot 5^{12} \cdot 11^{9} \cdot 13^{3} \cdot 41^{3} \cdot 43^{3} \cdot 97 \cdot 101^{2} \cdot 103^{3} \cdot 107^{4} \cdot 109^{5} \cdot 113^{6} \cdot 127^{10} . \\
& 131^{9} \cdot 137^{8} \cdot 139^{8} \cdot 149^{6} \cdot 151^{6} \cdot 157^{5} \cdot 163^{4} \cdot 167^{3} \cdot 173^{2} \cdot 179 \cdot 181 .
\end{aligned}
$$

The small prime factors are indicative of the fact that there is an underlying product formula. In fact for the (3,2)-case, the Hankel determinant is explicitly given by ([1], Theorem 4):

$$
H_{n}^{(3,2)}=\prod_{i=1}^{n} \frac{(6 i+4) !(2 i+1) !}{2(4 i+2) !(4 i+3) !} .
$$

We mention also the $(3,0)$-case for which the Hankel determinant also has small prime factors, and can be shown to possess the product evaluation [2]:

$$
H_{n}^{(3,0)}=\prod_{i=1}^{n} \frac{3(3 i+1)(6 i) !(2 i) !}{(4 i) !(4 i+1) !} .
$$

We will say more about this evaluation as the first example in Section 8.

For the $(3,1)$-case, we get the following intriguing evaluations:

$$
\begin{aligned}
H_{10}^{(3,1)=} & 2^{2} \cdot 7^{2} \cdot 37 \cdot 41^{2} \cdot 43^{3} \cdot 47^{2} \cdot 53 \cdot 41740796329 \\
H_{20}^{(3,1)=} & 3^{8} \cdot 29 \cdot 67 \cdot 71^{2} \cdot 73^{3} \cdot 79^{5} \cdot 83^{6} \cdot 89^{5} \cdot 97^{4} \cdot 101^{3} \cdot 103^{3} \cdot 107^{2} \cdot 109^{2} . \\
& 113 \cdot 631 \cdot 548377971864917477341 \\
H_{30}^{(3,1)}= & 2^{10} \cdot 5^{10} \cdot 11^{9} \cdot 13^{2} \cdot 41^{3} \cdot 43^{2} \cdot 97 \cdot 101^{2} \cdot 103^{3} \cdot 107^{4} \cdot 109^{5} \cdot 113^{6} . \\
& 127^{9} \cdot 131^{8} \cdot 137^{7} \cdot 139^{7} \cdot 149^{5} \cdot 151^{5} \cdot 157^{4} \cdot 163^{3} \cdot 167^{2} \cdot 173 \cdot 569 . \\
& 920397320923 \cdot 56029201596264233691799 .
\end{aligned}
$$

The existence of large primes in the factorizations indicates that $H_{n}^{(3,1)}$ does not have a product form evaluation. However if we write $H_{n}=P_{n} Q_{n}$ where $P_{n}$ is the product of 
the small primes and $Q_{n}$ is the product of the large primes left over, then it appears that the estimates $\log P_{n}=\Omega\left(n^{2}\right)$ and $\log Q_{n}=O(n)$ hold. This suggests that these Hankel determinants can be represented as a sum of $O(n)$ number of products, all of which have very similar representations.

The purpose of this paper is to provide a method that evaluates $H_{n}^{(3,1)}$ and a number of other Hankel determinants as almost products. For $H_{n}=H_{n}^{(3,1)}$, we obtain

$$
H_{n}=(-1)^{n} \prod_{i=1}^{n} \frac{(6 i-3) !(3 i+2) !(2 i-1) !}{(4 i-1) !(4 i+1) !(3 i-2) !} \sum_{i=0}^{n} \frac{n !(3 n+i+2) !(-6)^{i}}{(3 n+2) !(n-i) !(2 i+1) !}
$$

or alternately

$$
H_{n}=\prod_{i=1}^{n} \frac{(6 i+4) !(2 i+1) !}{2(4 i+2) !(4 i+3) !} \sum_{i=0}^{n} \frac{n !(4 n+3) ! !(3 n+i+2) !}{(3 n+2) ! i !(n-i) !(4 n+2 i+3) ! !}
$$

each as a sum of $n+1$ products. The method actually evaluates more, and we describe now the general situation in the $(3,1)$-case, which consists of three basic ingredients:

(I) Replace $a_{k}$ with polynomials

$$
a_{k}(x)=a_{k}^{(3,1)}(x)=\sum_{m=0}^{k}\left(\begin{array}{c}
3 k+1-m \\
k-m
\end{array}\right) x^{m}
$$

so that $a_{k}(x)$ is a monic polynomial of degree $k$ with $a_{k}=a_{k}(0)$.

(II) Show that the $a_{k}(x)$ satisfy certain differential-convolution equations.

(III) Show that the resulting determinants $H_{n}(x)$ themselves satisfy certain differential equations.

The $(n+1) \times(n+1)$ Hankel determinant $H_{n}(x)=H_{n}^{(3,1)}(x)$ is then expressed as the power series solution of the differential equation in (III), and we give it here as it is stated as Theorem 2:

$$
H_{n}(x)=(-1)^{n} \prod_{i=1}^{n} \frac{(6 i-3) !(3 i+2) !(2 i-1) !}{(4 i-1) !(4 i+1) !(3 i-2) !} \sum_{i=0}^{n} \frac{n !(3 n+i+2) ! 2^{i}(x-3)^{i}}{(3 n+2) !(n-i) !(2 i+1) !} .
$$

An alternate expression for this evaluation appears in Theorem 10 in Section 8.

Similarly, steps (I), (II), (III), mutatis mutandis, yield (Theorem 5):

$$
H_{n}^{(2,1)}(x)=(-1)^{n}(2 n+1) \sum_{i=0}^{n} \frac{(n+i) ! 2^{i}(x-2)^{i}}{(n-i) !(2 i+1) !}
$$

where

$$
a_{k}(x)=a_{k}^{(2,1)}(x)=\sum_{m=0}^{k}\left(\begin{array}{c}
2 k+1-m \\
k-m
\end{array}\right) x^{m}
$$


These polynomial families have a number of interesting properties that we briefly discuss. For example, the polynomials $H_{n}^{(3,1)}(x)$ satisfy a three-term recursion, their roots are real, and interlace. Furthermore the specializations at $x=3, \frac{3}{2}, \frac{3}{4}$ all have product evaluations. The polynomials $H_{n}^{(2,1)}(x)$ form an orthogonal family. A few other classes of Hankel determinants can be evaluated in almost product form by simple transformations of these polynomials (e.g., Example 8 in Section 8, and Corollaries 7 and 8 in Section $6.2)$.

Returning briefly to the general case of the determinants $H_{n}^{(\beta, \alpha)}$, we remark that there are a few more cases which fall under the method described above. These would also include the same three ingredients:

(I) Replace $a_{k}$ with polynomials

$$
a_{k}(x)=a_{k}^{(\beta, \alpha)}(x)=\sum_{m=0}^{k}\left(\begin{array}{c}
\beta k+\alpha-m \\
k-m
\end{array}\right) x^{m}
$$

so that $a_{k}(x)$ is a monic polynomial of degree $k$ with $a_{k}=a_{k}(0)$.

(II) Show that the $a_{k}(x)$ satisfy certain differential-convolution equations.

(III) Show that the resulting determinants $H_{n}(x)$ themselves satisfy certain differential equations.

The $(3,1)$-case and the $(2,1)$-case are governed by second order differential equations, but even these cases already present considerable technical problems to overcome. We mention some further difficulties that arise in the consideration of other $(\beta, \alpha)$-cases in Section 7. A number of additional almost product evaluations of Hankel determinants are given in Section 8. For these additional results given as Theorems 6, 7, 8, 9, 10 and special product form evaluations that appear in (168), (169) and (172), we provide the necessary identities for proving the differential equations, mimicking the proofs we present for $H_{n}^{(3,1)}(x)$ and $H_{n}^{(2,1)}(x)$.

Finally, a remarkable property of these $(n+1) \times(n+1)$ Hankel determinants $H_{n}^{(\beta, \alpha)}(x)$ is that the degree of the polynomial $H_{n}(x)$ is only $n$, indicating an extraordinary amount of cancellation in the expansion of the determinant. The unusual degree of cancellation is a basic property of a large class of Hankel determinants with polynomial entries. This class contains the Hankel determinants defined by polynomials in (10) that we consider. The degree result is of independent interest, and we include an exact statement and a proof of it as Theorem 11 in Appendix III.

We would like to remark that the differential-convolution equations (II) used in this paper are reminiscent of the equations that arise in the study of the Painlevé II equation and the Toda lattice $[6,5]$. 


\section{The $(3,1)$-case}

\subsection{Differential-convolution equations}

In the proof of the $(3,1)$-case, we denote the polynomials $a_{k}^{(3,1)}(x)$ by $a_{k}, H_{n}^{(3,1)}(x)$ by $H_{n}$, and the differentiation operator by $d_{x}$.

We need two identities given below in Lemmas 1 and 2. The first is a differentialconvolution equation. The second identity involves convolutions and $a_{k}$ but no derivatives. The proofs of these two lemmas are given in Appendix II.

Lemma 1 Let the polynomials $a_{k}=a_{k}^{(3,1)}(x)$ be as defined in (7). Then

$$
\begin{aligned}
(x-3)(2 x-3)(4 x-3) d_{x} a_{k}= & 2(2 k+3) a_{k+1}-\left(8 x^{2}-18 x+27 k+36\right) a_{k} \\
& +4\left(2 x^{2}-6 x+3\right) c_{k}-27\left(2 x^{2}-6 x+3\right) c_{k-1}
\end{aligned}
$$

where

$$
c_{k}=c_{k}(x)=\sum_{m=0}^{k} a_{m}(x) a_{k-m}(x), \quad\left(c_{-1}=0\right)
$$

Lemma 2 With $a_{k}=a_{k}^{(3,1)}(x)$ and $c_{k}=c_{k}(x)$ as in Lemma 1, we have

$$
\begin{aligned}
& 4(2 k+5)(x-1) a_{k+2} \\
& -\left(2\left(16 x^{3}-72 x^{2}+135 x-81\right) k+2\left(24 x^{3}-92 x^{2}+180 x-117\right)\right) a_{k+1} \\
& +\left(27(2 x-3)^{3} k+54(2 x-3)\left(2 x^{2}-4 x+3\right)\right) a_{k}+8(x-1)\left(2 x^{2}-6 x+3\right) c_{k+1} \\
& +2\left(8 x^{4}-114 x^{3}+324 x^{2}-297 x+81\right) c_{k}-27 x(2 x-3)\left(2 x^{2}-12 x+9\right) c_{k-1}=0 .
\end{aligned}
$$

Lemmas 1 and 2 will be needed for the proof of the differential equation satisfied by the determinants $H_{n}$. This differential equation is given below in Theorem 1 .

In addition to the first two identities in Lemma 1 and 2, a much more complicated third identity involving the $a_{k}$ is also needed for the proof of this differential equation. This will emerge in the course of the proof of (14).

Theorem 1 Let the polynomials $a_{k}=a_{k}^{(3,1)}(x)$ be as in (7) and define the $(n+1) \times(n+1)$ Hankel matrix by

$$
A_{n}=A_{n}(x)=\left[a_{i+j}(x)\right]_{0 \leq i, j \leq n}
$$

Then

$$
H_{n}=H_{n}^{(3,1)}(x)=\operatorname{det} A_{n}(x)
$$

satisfies the differential equation

$$
(x-1)(x-3) d_{x}^{2} y+(2(n+2)(x-3)+3) d_{x} y-3 n(n+1) y=0 .
$$


Proof This is easy to check for $n=0,1$. Henceforth we assume that $n \geq 2$.

We will find an expression for the first derivative $d_{x} H_{n}$ in Section 2.2. An expression for the second derivative $d_{x}^{2} H_{n}$ is developed in Sections 2.3 and 2.4. This is followed by Section 3 on specializations of $x$ : product formulas for $H_{n}(3), H_{n}\left(\frac{3}{2}\right)$ and $H_{n}\left(\frac{3}{4}\right)$ are given as three corollaries in Sections 3.1, 3.2, and 3.3. The specializations make use of a Dodgson-like expansion result we prove as Proposition 1 at the start of Section 3. The proof of Theorem 1 continues in Section 4 where we put together the expressions obtained for the derivatives and the third identity mentioned to prove (14).

\subsection{Calculating the first derivative}

The first step is to find a reasonably simple form for the derivative of $H_{n}$. We begin with the expression

$$
d_{x} H_{n}=\operatorname{Tr}\left(A_{n}^{-1} d_{x} A_{n}\right) H_{n}
$$

for the derivative of a determinant, where

$$
d_{x} A_{n}=d_{x} A_{n}(x)=\left[d_{x} a_{i+j}(x)\right]_{0 \leq i, j \leq n} .
$$

Referring to Lemma 1, we write

$$
(x-3)(2 x-3)(4 x-3) \operatorname{Tr}\left(A_{n}^{-1} d_{x} A_{n}\right)
$$

as

$$
\begin{aligned}
& 2 \operatorname{Tr}\left(A_{n}^{-1}\left[(2(i+j)+3) a_{i+j+1}\right]_{0 \leq i, j \leq n}\right) \\
+ & \operatorname{Tr}\left(A_{n}^{-1}\left[-\left(8 x^{2}-18 x+27(i+j)+36\right) a_{i+j}\right]_{0 \leq i, j \leq n}\right) \\
+ & 4\left(2 x^{2}-6 x+3\right) \operatorname{Tr}\left(A_{n}^{-1}\left[c_{i+j}\right]_{0 \leq i, j \leq n}\right) \\
- & 27\left(2 x^{2}-6 x+3\right) \operatorname{Tr}\left(A_{n}^{-1}\left[c_{i+j-1}\right]_{0 \leq i, j \leq n}\right)
\end{aligned}
$$

where the convolutions $c_{k}$ are defined in (12).

We render each of these four expressions (16)-(19) in a simple form, and then combine them all into an expression for the derivative in (15). After that is done, we go through a similar computation for the second derivative of $H_{n}$, where we use the recursion in Lemma 2 for the simplifications. The differential equation will follow from a third identity, the proof of which makes up the bulk of the work for the rest of the argument.

We begin with the trace term (16): Let I denote the identity matrix of relevant dimension and define the two matrices

$$
B_{n}=B_{n}(x)=\left[\begin{array}{cccc}
a_{1} & a_{2} & \ldots & a_{n+1} \\
a_{2} & a_{3} & \ldots & a_{n+2} \\
\vdots & & & \vdots \\
a_{n+1} & a_{n+2} & \ldots & a_{2 n+1}
\end{array}\right]
$$




$$
L_{n}=\left[\begin{array}{lllll}
0 & & & & \\
& 1 & & & \\
& & 2 & & \\
& & & \ddots & \\
& & & & n
\end{array}\right]
$$

Then

$2 \operatorname{Tr}\left(A_{n}^{-1}\left[(2(i+j)+3) a_{i+j+1}(x)\right]_{0 \leq i, j \leq n}\right)=2 \operatorname{Tr}\left(A_{n}^{-1}\left(\left(2 L_{n}+\frac{3}{2} I\right) B_{n}+B_{n}\left(2 L_{n}+\frac{3}{2} I\right)\right)\right)$.

Now define $\sigma_{0}, \sigma_{1}, \ldots, \sigma_{n}$ and $K_{n}$ as follows:

$$
\left[\begin{array}{c}
\sigma_{0} \\
\sigma_{1} \\
\vdots \\
\sigma_{n}
\end{array}\right]=A_{n}^{-1}\left[\begin{array}{c}
a_{n+1} \\
a_{n+2} \\
\vdots \\
a_{2 n+1}
\end{array}\right]
$$

and

$$
K_{n}=\operatorname{det}\left[\begin{array}{ccccc}
a_{0} & a_{1} & \ldots & a_{n-1} & a_{n+1} \\
a_{1} & a_{2} & \ldots & a_{n} & a_{n+2} \\
\vdots & & \ddots & & \\
a_{n-1} & a_{n} & \ldots & a_{2 n-2} & a_{2 n} \\
a_{n} & a_{n+1} & \ldots & a_{2 n-1} & a_{2 n+1}
\end{array}\right] .
$$

By Cramer's rule we have

$$
\sigma_{n}=\frac{K_{n}}{H_{n}}
$$

Therefore

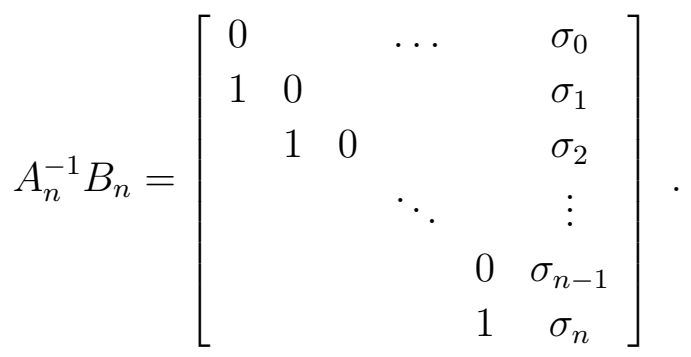

and

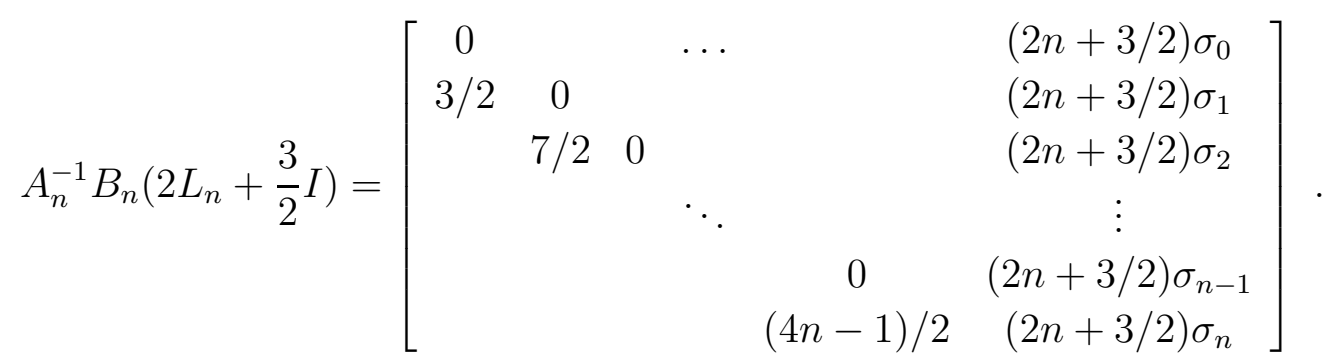


Since

$$
\left(2 L_{n}+\frac{3}{2} I\right) B_{n} A_{n}^{-1}=\left(A_{n}^{-1} B_{n}\left(2 L_{n}+\frac{3}{2} I\right)\right)^{T}
$$

we can write

$$
\begin{aligned}
2 \operatorname{Tr} & \left(A_{n}^{-1}\left(\left(2 L_{n}+\frac{3}{2} I\right) B_{n}+B_{n}\left(2 L_{n}+\frac{3}{2} I\right)\right)\right) \\
& =2 \operatorname{Tr}\left(A_{n}^{-1}\left(2 L_{n}+\frac{3}{2} I\right) B_{n}\right)+2 \operatorname{Tr}\left(A_{n}^{-1} B_{n}\left(2 L_{n}+\frac{3}{2} I\right)\right) \\
& =2 \operatorname{Tr}\left(\left(2 L_{n}+\frac{3}{2} I\right) B_{n} A_{n}^{-1}\right)+2 \operatorname{Tr}\left(A_{n}^{-1} B_{n}\left(2 L_{n}+\frac{3}{2} I\right)\right) \\
& =4 \operatorname{Tr}\left(A_{n}^{-1} B_{n}\left(2 L_{n}+\frac{3}{2} I\right)\right) \\
& =4\left(2 n+\frac{3}{2}\right) \sigma_{n} \\
& =2(4 n+3) \sigma_{n} .
\end{aligned}
$$

The last expression gives

$$
2 \operatorname{Tr}\left(A_{n}^{-1}\left[(2(i+j)+3) a_{i+j+1}\right]_{0 \leq i, j \leq n}\right)=2(4 n+3) \frac{K_{n}}{H_{n}}
$$

for the desired form of the first term (16). It is useful to record in passing that

$$
\frac{K_{n}}{H_{n}}=\sigma_{n}=\operatorname{Tr}\left(A_{n}^{-1} B_{n}\right) .
$$

The identity (25) will be useful later when we calculate the derivative of $K_{n}$.

Now we consider the second trace term (17). The calculation of this term is done in the same manner as the first term but the evaluation is somewhat simpler. We get the expression:

$$
\operatorname{Tr}\left(A_{n}^{-1}\left[-\left(8 x^{2}-18 x+27(i+j)+35\right) a_{i+j}\right]_{0 \leq i, j \leq n}\right)=-(n+1)\left(8 x^{2}-18 x+27 n+36\right) .
$$

The final two terms (18) and (19) require a new technique. We will use ideas from $[6,5]$ where an identity similar to the following is used:

$$
\left[c_{i+j}\right]_{0 \leq i, j \leq n}=E_{n} A_{n}+A_{n} E_{n}^{T}
$$

where

$$
E_{n}=E_{n}(x)=\left[\begin{array}{ccccc}
a_{0} / 2 & 0 & & & \\
a_{1} / 2 & a_{0} & & & \\
a_{2} / 2 & a_{1} & a_{0} & & \\
\vdots & & & \ddots & \\
a_{n} / 2 & a_{n-1} & a_{n-2} & & a_{0}
\end{array}\right]
$$


Note that the first column of $E_{n}$ is divided by two. This allows for the immediate computation

$$
\operatorname{Tr}\left(A_{n}^{-1}\left[c_{i+j}\right]_{0 \leq i, j \leq n}\right)=(2 n+1) a_{0} .
$$

So the third term (18) is

$$
4\left(2 x^{2}-6 x+3\right) \operatorname{Tr}\left(A_{n}^{-1}\left[c_{i+j}\right]_{0 \leq i, j \leq n}\right)=4(2 n+1)\left(2 x^{2}-6 x+3\right) .
$$

The trace term (19) that involves

$$
\left[c_{i+j-1}\right]_{0 \leq i, j \leq n}
$$

is handled in a similar way. We have the equation

$$
\left[c_{i+j-1}\right]_{0 \leq i, j \leq n}=F_{n} A_{n}+A_{n} F_{n}^{T}
$$

where

$$
F_{n}=F_{n}(x)=\left[\begin{array}{ccccc}
0 & & & & \\
a_{0} & 0 & & & \\
a_{1} & a_{0} & 0 & & \\
\vdots & & \ddots & & \\
a_{n-1} & a_{n-2} & \ldots & a_{0} & 0
\end{array}\right]
$$

The identity (30) leads to the computation

$$
\operatorname{Tr}\left(A_{n}^{-1}\left[c_{i+j-1}\right]_{0 \leq i, j \leq n}\right)=0
$$

so that the trace term (19) evaluates to zero:

$$
-27\left(2 x^{2}-6 x+3\right) \operatorname{Tr}\left(A_{n}^{-1}\left[c_{i+j-1}\right]_{0 \leq i, j \leq n}\right)=0 .
$$

Adding the expressions (24), (26), (29), (32) and multiplying through by $H_{n}$ we obtain the following expression for the first derivative

\section{Lemma 3}

$$
\begin{aligned}
(x-3)(2 x-3)(4 x-3) d_{x} H_{n}= & \left(8 n x^{2}-6(5 n+1) x-3\left(9 n^{2}+13 n+8\right)\right) H_{n} \\
& +2(4 n+3) K_{n} .
\end{aligned}
$$

\subsection{Preparatory work for the second derivative}

We state and prove a lemma which is preparatory to the calculation of the second derivative of $H_{n}$. First define two new determinants as follows:

$$
M_{n}=M_{n}(x)=\operatorname{det}\left[\begin{array}{cccccc}
a_{0} & a_{1} & \ldots & a_{n-2} & a_{n+1} & a_{n} \\
a_{1} & a_{2} & \ldots & a_{n-1} & a_{n+2} & a_{n+1} \\
\vdots & & \ddots & & & \\
a_{n-1} & a_{n} & \ldots & a_{2 n-3} & a_{2 n} & a_{2 n-1} \\
a_{n} & a_{n+1} & \ldots & a_{2 n-2} & a_{2 n+1} & a_{2 n}
\end{array}\right]
$$




$$
N_{n}=N_{n}(x)=\operatorname{det}\left[\begin{array}{cccccc}
a_{0} & a_{1} & \ldots & a_{n-2} & a_{n-1} & a_{n+2} \\
a_{1} & a_{2} & \ldots & a_{n-1} & a_{n} & a_{n+3} \\
\vdots & & \ddots & & & \\
a_{n-1} & a_{n} & \ldots & a_{2 n-3} & a_{2 n-2} & a_{2 n+1} \\
a_{n} & a_{n+1} & \ldots & a_{2 n-2} & a_{2 n-1} & a_{2 n+2}
\end{array}\right]
$$

where $a_{k}=a_{k}(x)$ are the polynomials defined in (7). Then there is a linear relationship between the four determinants $H_{n}, K_{n}, M_{n}$, and $N_{n}$ as stated in the following lemma.

\section{Lemma 4}

$$
\begin{aligned}
& 4(4 n+5)(x-1) N_{n}+4(4 n+1)(x-1) M_{n} \\
+ & \left(-16(4 n+1) x^{3}+8(36 n+7) x^{2}-108(5 n+2) x+6(54 n+31)\right) K_{n} \\
+ & \left((64 n+16) x^{4}+\left(216 n^{2}-24 n-12\right) x^{3}-\left(972 n^{2}+800 n+108\right) x^{2}\right. \\
& \left.\quad+\left(1458 n^{2}+1770 n+378\right) x-729 n^{2}-1083 n-324\right) H_{n}=0 .
\end{aligned}
$$

Proof of the Lemma: First we make use of the recursion in Lemma 2 for each index $k$. Putting these all together in matrix form, we apply the operator

$$
\operatorname{Tr}\left(A_{n}^{-1} *\right)
$$

to obtain the trace identity

$$
\begin{aligned}
& \operatorname{Tr}\left(A_{n}^{-1}\left[4(2(i+j)+5)(x-1) a_{i+j+2}\right]_{0 \leq i, j \leq n}\right) \\
& \quad+\operatorname{Tr}\left(A _ { n } ^ { - 1 } \left[\left(-2\left(16 x^{3}-72 x^{2}+135 x-81\right)(i+j)\right.\right.\right. \\
& \left.\left.\left.\quad-2\left(24 x^{3}-92 x^{2}+180 x-117\right)\right) a_{i+j+1}\right]_{0 \leq i, j \leq n}\right) \\
& \quad+\operatorname{Tr}\left(A_{n}^{-1}\left[\left(27(2 x-3)^{3}(i+j)+54(2 x-3)\left(2 x^{2}-4 x+3\right)\right) a_{i+j}\right]_{0 \leq i, j \leq n}\right) \\
& \quad+\operatorname{Tr}\left(A_{n}^{-1}\left[8(x-1)\left(2 x^{2}-6 x+3\right) c_{i+j+1}\right]_{0 \leq i, j \leq n}\right) \\
& \quad+\operatorname{Tr}\left(A_{n}^{-1}\left[2\left(8 x^{4}-114 x^{3}+324 x^{2}-297 x+81\right) c_{i+j}\right]_{0 \leq i, j \leq n}\right) \\
& \quad+\operatorname{Tr}\left(A_{n}^{-1}\left[-27 x(2 x-3)\left(2 x^{2}-12 x+9\right) c_{i+j-1}\right]_{0 \leq i, j \leq n}\right)=0 .
\end{aligned}
$$

Each of these six traces (37)-(42) is calculated in a similar manner as was done above in the calculation of the first derivative of $H_{n}$. The first and fourth trace will involve a small extension to what was used above. We will start with the computation of (37). As before we write this as

$$
4(x-1) \operatorname{Tr}\left(A_{n}^{-1}\left(\left(2 L_{n}+\frac{5}{2} I\right)\left[a_{i+j+2}\right]_{0 \leq i, j \leq n}+\left[a_{i+j+2}\right]_{0 \leq i, j \leq n}\left(2 L_{n}+\frac{5}{2} I\right)\right)\right) .
$$

Using the fact that

$$
\begin{aligned}
& 4(x-1) \operatorname{Tr}\left(A_{n}^{-1}\left(2 L_{n}+\frac{5}{2} I\right)\left[a_{i+j+2}\right]_{0 \leq i, j \leq n}\right) \\
& \quad=4(x-1) \operatorname{Tr}\left(\left(2 L_{n}+\frac{5}{2} I\right)\left[a_{i+j+2}\right]_{0 \leq i, j \leq n} A_{n}^{-1}\right)
\end{aligned}
$$


and

$$
\left(\left(2 L_{n}+\frac{5}{2} I\right)\left[a_{i+j+2}\right]_{0 \leq i, j \leq n} A_{n}^{-1}\right)^{T}=A_{n}^{-1}\left[a_{i+j+2}\right]_{0 \leq i, j \leq n}\left(2 L_{n}+\frac{5}{2} I\right)
$$

we see that we can write (43) as

$$
8(x-1) \operatorname{Tr}\left(A_{n}^{-1}\left[a_{i+j+2}\right]_{0 \leq i, j \leq n}\left(2 L_{n}+\frac{5}{2} I\right)\right) .
$$

We will obtain a representation of the trace above in terms of $H_{n}, M_{n}$, and $N_{n}$. Introduce $\tau_{0}, \tau_{1}, \ldots, \tau_{n}$ as follows:

$$
\left[\begin{array}{c}
\tau_{0} \\
\tau_{1} \\
\vdots \\
\tau_{n}
\end{array}\right]=A_{n}^{-1}\left[\begin{array}{c}
a_{n+2} \\
a_{n+3} \\
\vdots \\
a_{2 n+2}
\end{array}\right]
$$

As before we observe that

$$
A_{n}^{-1}\left[a_{i+j+2}\right]_{0 \leq i, j \leq n}=\left[\begin{array}{cccccccc}
0 & & & & & & \sigma_{0} & \tau_{0} \\
0 & 0 & & & & & \sigma_{1} & \tau_{1} \\
1 & 0 & \ddots & & & & \sigma_{2} & \tau_{2} \\
& 1 & & \ddots & & & \sigma_{3} & \tau_{3} \\
& & \ddots & & \ddots & & \vdots & \vdots \\
& & & \ddots & & 0 & \sigma_{n-2} & \tau_{n-2} \\
& & & & \ddots & 0 & \sigma_{n-1} & \tau_{n-1} \\
& & & & 1 & \sigma_{n} & \tau_{n}
\end{array}\right]
$$

and therefore

$$
A_{n}^{-1}\left[a_{i+j+2}\right]_{0 \leq i, j \leq n}\left(2 L_{n}+\frac{5}{2} I\right)
$$

expands to

$$
\left[\begin{array}{ccccccc}
0 & & & & & (4 n+1) \sigma_{0} / 2 & (4 n+5) \tau_{0} / 2 \\
0 & \ddots & & & & (4 n+1) \sigma_{1} / 2 & (4 n+5) \tau_{1} / 2 \\
5 / 2 & & \ddots & & & (4 n+1) \sigma_{2} / 2 & (4 n+5) \tau_{2} / 2 \\
& \ddots & & \ddots & & \vdots & \vdots \\
& & \ddots & & 0 & (4 n+1) \sigma_{n-2} / 2 & (4 n+5) \tau_{n-2} / 2 \\
& & & \ddots & 0 & (4 n+1) \sigma_{n-1} / 2 & (4 n+5) \tau_{n-1} / 2 \\
& & & & (4 n-3) / 2 & (4 n+1) \sigma_{n} / 2 & (4 n+5) \tau_{n} / 2
\end{array}\right] .
$$

Thus (44) simplifies to

$$
4(x-1)\left((4 n+1) \sigma_{n-1}+(4 n+5) \tau_{n}\right) .
$$


By Cramer's rule we have

$$
\begin{aligned}
\sigma_{n-1} & =\frac{M_{n}}{H_{n}} \\
\tau_{n} & =\frac{N_{n}}{H_{n}}
\end{aligned}
$$

so, in summary, the first trace (37) evaluates to

$$
4(x-1)\left((4 n+1) \frac{M_{n}}{H_{n}}+(4 n+5) \frac{N_{n}}{H_{n}}\right) .
$$

Now we consider the second trace (38). The evaluation of this trace proceeds exactly as the evaluation of the trace (16) in the computation of the derivative of $H_{n}$. (38) evaluates to

$$
-2\left(2\left(16 x^{3}-72 x^{2}+135 x-81\right) n+\left(24 x^{3}-92 x^{2}+180 x-117\right)\right) \frac{K_{n}}{H_{n}} .
$$

Similarly, the third trace (39) evaluates to

$$
(n+1)\left(27(2 x-3)^{3} n+54(2 x-3)\left(2 x^{2}-4 x+3\right)\right) .
$$

The next trace (40) requires that we define a matrix $G_{n}$ in a similar manner to $E_{n}$ and $F_{n}$. Specifically we define

$$
G_{n}=G_{n}(x)=\left[\begin{array}{cccccc}
a_{1} / 2 & a_{0} / 2 & & & \\
a_{2} / 2 & a_{1} / 2 & a_{0} & & & \\
a_{3} / 2 & a_{2} / 2 & a_{1} & a_{0} & & \\
\vdots & \vdots & & & \ddots & \\
a_{n} / 2 & a_{n-1} / 2 & a_{n-2} & \ldots & & a_{0} \\
a_{n+1} / 2 & a_{n} / 2 & a_{n-1} & \ldots & & a_{1}
\end{array}\right] .
$$

With this definition of $G_{n}$ we can write

$$
\left[c_{i+j+1}\right]_{0 \leq i, j \leq n}=G_{n} A_{n}+A_{n} G_{n}^{T}+a_{0}\left[\begin{array}{cccc}
0 & & \ldots & 0 \\
\vdots & & & \vdots \\
0 & 0 & \ldots & 0 \\
a_{n+1} & a_{n+2} & \ldots & a_{2 n+1}
\end{array}\right]+a_{0}\left[\begin{array}{cccc}
0 & \ldots & 0 & a_{n+1} \\
\vdots & & 0 & a_{n+2} \\
& & \vdots \\
0 & \ldots & 0 & a_{2 n+1}
\end{array}\right] .
$$

We have

$$
A_{n}^{-1}\left[\begin{array}{cccc}
0 & \ldots & 0 & a_{n+1} \\
\vdots & & 0 & a_{n+2} \\
& & & \vdots \\
0 & \ldots & 0 & a_{2 n+1}
\end{array}\right]=\left[\begin{array}{cccc}
0 & \ldots & 0 & \sigma_{0} \\
\vdots & & 0 & \sigma_{1} \\
& & & \vdots \\
0 & \ldots & 0 & \sigma_{n}
\end{array}\right]
$$


where $\sigma_{i}$ is as defined in (20). It follows that

$$
\operatorname{Tr}\left(A_{n}^{-1}\left[\begin{array}{cccc}
0 & \ldots & 0 & a_{n+1} \\
\vdots & & 0 & a_{n+2} \\
& & & \vdots \\
0 & \ldots & 0 & a_{2 n+1}
\end{array}\right]\right)=\sigma_{n}
$$

The term

$$
\operatorname{Tr}\left(A_{n}^{-1}\left[\begin{array}{cccc}
0 & & \ldots & 0 \\
\vdots & & & \vdots \\
0 & 0 & \ldots & 0 \\
a_{n+1} & a_{n+2} & \ldots & a_{2 n+1}
\end{array}\right]\right)
$$

also comes out to $\sigma_{n}$ because

$$
\left(\left[\begin{array}{cccc}
0 & & \ldots & 0 \\
\vdots & & & \vdots \\
0 & 0 & \ldots & 0 \\
a_{n+1} & a_{n+2} & \ldots & a_{2 n+1}
\end{array}\right] A_{n}^{-1}\right)^{T}=A_{n}^{-1}\left[\begin{array}{cccc}
0 & \ldots & 0 & a_{n+1} \\
\vdots & & 0 & a_{n+2} \\
& & & \vdots \\
0 & \ldots & 0 & a_{2 n+1}
\end{array}\right] .
$$

Putting all this together we see that the fourth trace (40) evaluates to

$$
16(x-1)\left(2 x^{2}-6 x+3\right)\left(n(x+4)+\frac{K_{n}}{H_{n}}\right) .
$$

The evaluation of the fifth and sixth traces (41) and (42) are done in exactly the same manner as the traces (18) and (19). For the fifth trace (41) we obtain

$$
\begin{aligned}
\operatorname{Tr}\left(A _ { n } ^ { - 1 } \left[2 \left(8 x^{4}-114 x^{3}+\right.\right.\right. & \left.\left.\left.324 x^{2}-297 x+81\right) c_{i+j}\right]_{0 \leq i, j \leq n}\right) \\
= & 2(2 n+1)\left(8 x^{4}-114 x^{3}+324 x^{2}-297 x+81\right)
\end{aligned}
$$

and the sixth trace (42) evaluates to zero just as the trace in (19):

$$
\operatorname{Tr}\left(A_{n}^{-1}\left[-27 x(2 x-3)\left(2 x^{2}-12 x+9\right) c_{i+j-1}\right]_{0 \leq i, j \leq n}\right)=0 .
$$

Adding the expressions we have found for the six traces in (47)-(52) we obtain the identity in (36). This finishes the proof of Lemma 4.

\subsection{Calculating the second derivative}

We are now ready to calculate the second derivative of $H_{n}$. We begin with equation (33) for the derivative. The first step is to replace $K_{n}$ in equation (33) with the representation of $K_{n}$ as a trace from (25). Inserting this expression into equation (33) we get

$$
\begin{aligned}
(x-3)(2 x-3)(4 x-3) d_{x} H_{n}= & \left(8 n x^{2}-6(5 n+1) x-3\left(9 n^{2}+13 n+8\right)\right) H_{n} \\
& +2(4 n+3) \operatorname{Tr}\left(A_{n}^{-1} B_{n}\right) H_{n} .
\end{aligned}
$$


In order to obtain the second derivative of $H_{n}$, we differentiate (53). We obtain

$$
\begin{array}{r}
(x-3)(2 x-3)(4 x-3) d_{x}^{2} H_{n}+ \\
\left(8(n+3) x^{2}-6(5 n-13) x-3\left(9 n^{2}+13 n+29\right)\right) d_{x} H_{n}+ \\
2(8 n x-15 n-3) H_{n} \\
=2(4 n+3)\left(d_{x} \operatorname{Tr}\left(A_{n}^{-1} B_{n}\right)\right) H_{n}+2(4 n+3) \operatorname{Tr}\left(A_{n}^{-1} B_{n}\right) d_{x} H_{n} .
\end{array}
$$

Multiply both sides of the equation by

$$
(x-3)(2 x-3)(4 x-3)
$$

The second trace term on the right hand side now becomes

$$
2(4 n+3)(x-3)(2 x-3)(4 x-3) \operatorname{Tr}\left(A_{n}^{-1} B_{n}\right) d_{x} H_{n} .
$$

Using the identities (33) and (25), (54) can be written in terms of $H_{n}$ and $K_{n}$ as follows: as

$$
2(4 n+3)\left(\left(8 n x^{2}-6(5 n+1) x-3\left(9 n^{2}+13 n+8\right)\right) K_{n}+2(4 n+3) \frac{K_{n}^{2}}{H_{n}}\right) .
$$

Substituting back, we get

$$
\begin{array}{r}
(x-3)^{2}(2 x-3)^{2}(4 x-3)^{2} d_{x}^{2} H_{n}- \\
(x-3)(2 x-3)(4 x-3)\left(8(n-3) x^{2}-6(5 n-13) x-3\left(9 n^{2}+13 n+29\right)\right) d_{x} H_{n}- \\
2(x-3)(2 x-3)(4 x-3)(8 n x-15 n-3) H_{n}- \\
2(4 n+3)\left(8 n x^{2}-6(5 n+1) x-3\left(9 n^{2}+13 n+8\right)\right) K_{n}- \\
4(4 n+3)^{2} \frac{K_{n}^{2}}{H_{n}}- \\
2(4 n+3)(x-3)(2 x-3)(4 x-3)\left(d_{x} \operatorname{Tr}\left(A_{n}^{-1} B_{n}\right)\right) H_{n}=0
\end{array} .
$$

We will now focus on the simplification of the derivative of the trace

$$
(x-3)(2 x-3)(4 x-3)\left(d_{x} \operatorname{Tr}\left(A_{n}^{-1} B_{n}\right)\right)
$$

which is a factor of the last term on the left of equation (55). We use the fact that

$$
d_{x} A_{n}^{-1}=-A_{n}^{-1}\left(d_{x} A_{n}\right) A_{n}^{-1}
$$

and write

$$
d_{x} \operatorname{Tr}\left(A_{n}^{-1} B_{n}\right)=\operatorname{Tr}\left(A_{n}^{-1} d_{x} B_{n}\right)-\operatorname{Tr}\left(A_{n}^{-1}\left(d_{x} A_{n}\right) A_{n}^{-1} B_{n}\right) .
$$

Using equation (11) from Lemma 1 , and multiplying equation (56) by $(x-3)(2 x-$ $3)(4 x-3)$, we can write

$$
(x-3)(2 x-3)(4 x-3) \operatorname{Tr}\left(A_{n}^{-1} d_{x} B_{n}\right)
$$


as

$$
\begin{aligned}
& \operatorname{Tr}\left(A_{n}^{-1}\left[2(2(i+j+1)+3) a_{i+j+2}\right]_{0 \leq i, j \leq n}\right) \\
+ & \operatorname{Tr}\left(A_{n}^{-1}\left[-\left(8 x^{2}-18 x+27(i+j+1)+36\right) a_{i+j+1}\right]_{0 \leq i, j \leq n}\right) \\
+ & \operatorname{Tr}\left(A_{n}^{-1}\left[4\left(2 x^{2}-6 x+3\right) c_{i+j+1}\right]_{0 \leq i, j \leq n}\right) \\
+ & \operatorname{Tr}\left(A_{n}^{-1}\left[-27\left(2 x^{2}-6 x+3\right) c_{i+j}\right]_{0 \leq i, j \leq n}\right)
\end{aligned}
$$

and we can write

$$
(x-3)(2 x-3)(4 x-3) \operatorname{Tr}\left(A_{n}^{-1}\left(d_{x} A_{n}\right) A_{n}^{-1} B_{n}\right)
$$

as

$$
\begin{aligned}
& \operatorname{Tr}\left(A_{n}^{-1}\left[2(2(i+j)+3) a_{i+j+1}\right]_{0 \leq i, j \leq n} A_{n}^{-1} B_{n}\right) \\
+ & \operatorname{Tr}\left(A_{n}^{-1}\left[-\left(8 x^{2}-18 x+27(i+j)+36\right) a_{i+j}\right]_{0 \leq i, j \leq n} A_{n}^{-1} B_{n}\right) \\
+ & \operatorname{Tr}\left(A_{n}^{-1}\left[4\left(2 x^{2}-6 x+3\right) c_{i+j}\right]_{0 \leq i, j \leq n} A_{n}^{-1} B_{n}\right) \\
+ & \operatorname{Tr}\left(A_{n}^{-1}\left[-27\left(2 x^{2}-6 x+3\right) c_{i+j-1}\right]_{0 \leq i, j \leq n} A_{n}^{-1} B_{n}\right) .
\end{aligned}
$$

We have already evaluated traces that are similar to the first four terms (57)-(60) in the calculation of the trace terms in (16)-(19). In this case we obtain

$$
\begin{aligned}
& \operatorname{Tr}\left(A_{n}^{-1}\left[2(2(i+j+1)+3) a_{i+j+2}\right]_{0 \leq i, j \leq n}\right)=2\left((4 n+1) \frac{M_{n}}{H_{n}}+(4 n+5) \frac{N_{n}}{H_{n}}\right) \\
& \operatorname{Tr}\left(A_{n}^{-1}\left[-\left(8 x^{2}-18 x+27(i+j+1)+36\right) a_{i+j+1}\right]_{0 \leq i, j \leq n}\right) \\
& =-\left(8 x^{2}-18 x+54 n+63\right) \frac{K_{n}}{H_{n}} \\
& \operatorname{Tr}\left(A_{n}^{-1}\left[4\left(2 x^{2}-6 x+3\right) c_{i+j+1}\right]_{0 \leq i, j \leq n}\right)=\left(2 x^{2}-6 x+3\right)\left(8 n(x+4)+8 \frac{K_{n}}{H_{n}}\right) \\
& \operatorname{Tr}\left(A_{n}^{-1}\left[-27\left(2 x^{2}-6 x+3\right) c_{i+j}\right]_{0 \leq i, j \leq n}\right)=-27(2 n+1)\left(2 x^{2}-6 x+3\right)
\end{aligned}
$$

Next we evaluate the traces (61)-(64). To calculate the first of these, the trace in(61), we expand the matrix

$$
A_{n}^{-1}\left[2(2(i+j)+3) a_{i+j+1}\right]_{0 \leq i, j \leq n} A_{n}^{-1} B_{n}
$$

as

$$
A_{n}^{-1}\left(\left(4 L_{n}+3 I\right) B_{n}+B_{n}\left(4 L_{n}+3 I\right)\right) A_{n}^{-1} B_{n} .
$$

The trace (61) thus can be evaluated as

$$
\begin{aligned}
\operatorname{Tr}\left(\left(4 L_{n}+3 I\right) B_{n} A_{n}^{-1} B_{n} A_{n}^{-1}\right) & +\operatorname{Tr}\left(A_{n}^{-1} B_{n}\left(4 L_{n}+3 I\right) A_{n}^{-1} B_{n}\right) \\
& =2 \operatorname{Tr}\left(A_{n}^{-1} B_{n}\left(4 L_{n}+3 I\right) A_{n}^{-1} B_{n}\right) \\
& =2 \operatorname{Tr}\left(\left(4 L_{n}+3 I\right)\left(A_{n}^{-1} B_{n}\right)^{2}\right)
\end{aligned}
$$


where we used the symmetry of the matrices in the trace calculation. Using the expression (23) for $A_{n}^{-1} B_{n}$, we have

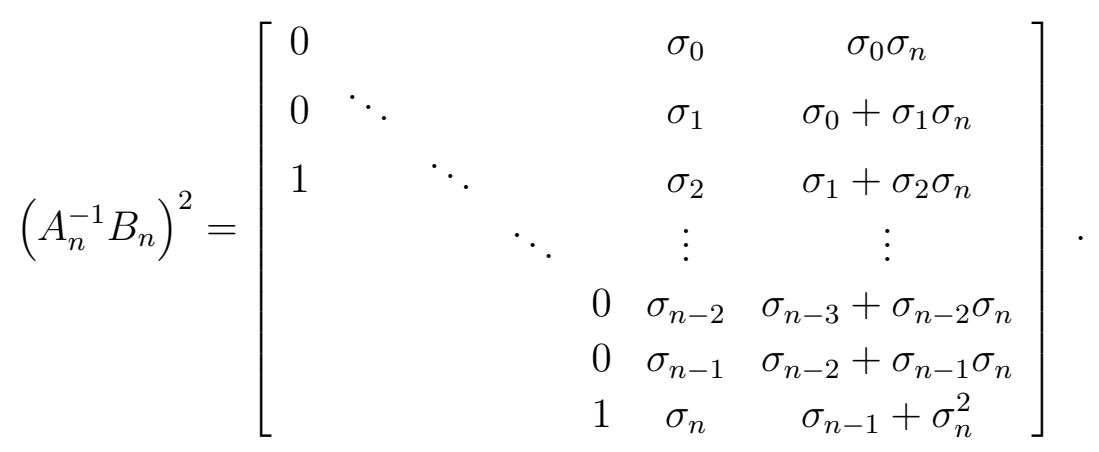

Therefore

$$
\left(4 L_{n}+3 I\right)\left(A_{n}^{-1} B_{n}\right)^{2}=\left[\begin{array}{ccccccc}
0 & & & & & 3 \sigma_{0} & 3 \sigma_{0} \sigma_{n} \\
0 & \ddots & & & & 7 \sigma_{1} & 7\left(\sigma_{0}+\sigma_{1} \sigma_{n}\right) \\
1 & & \ddots & & & 10 \sigma_{2} & 10\left(\sigma_{1}+\sigma_{2} \sigma_{n}\right) \\
& & & \ddots & & \vdots & \vdots \\
& & & & 0 & (4 n-5) \sigma_{n-2} & (4 n-5)\left(\sigma_{n-3}+\sigma_{n-2} \sigma_{n}\right) \\
& & & 0 & (4 n-1) \sigma_{n-1} & (4 n-1)\left(\sigma_{n-2}+\sigma_{n-1} \sigma_{n}\right) \\
& & & 1 & (4 n+3) \sigma_{n} & (4 n+3)\left(\sigma_{n-1}+\sigma_{n}^{2}\right)
\end{array}\right]
$$

and thus

$$
\begin{aligned}
\operatorname{Tr}\left(A_{n}^{-1}\left[2(2(i+j)+3) a_{i+j+1}\right]_{\leq i, j \leq n} A_{n}^{-1} B_{n}\right) & =2\left((4 n-1) \sigma_{n-1}+(4 n+3)\left(\sigma_{n-1}+\sigma_{n}^{2}\right)\right) \\
& =4(4 n+1) \frac{M_{n}}{H_{n}}+2(4 n+3) \frac{K_{n}^{2}}{H_{n}^{2}}
\end{aligned}
$$

where the last equality is a consequence of the identities (22) and (45).

Using similar reasoning, we see that the trace term (62)

$$
\operatorname{Tr}\left(A_{n}^{-1}\left[-\left(8 x^{2}-18 x+27(i+j)+36\right) a_{i+j}\right]_{0 \leq i, j \leq n} A_{n}^{-1} B_{n}\right)
$$

evaluates to

$$
-\left(8 x^{2}-18 x+54 n+36\right) \frac{K_{n}}{H_{n}} .
$$

The trace term (63) is expanded using (27). We obtain the following trace identities

$$
\begin{aligned}
\operatorname{Tr}\left(A _ { n } ^ { - 1 } \left[4 \left(2 x^{2}-6 x\right.\right.\right. & \left.\left.+3) c_{i+j}\right]_{0 \leq i, j \leq n} A_{n}^{-1} B_{n}\right) \\
& =4\left(2 x^{2}-6 x+3\right) \operatorname{Tr}\left(A_{n}^{-1}\left(E_{n} A_{n}+A_{n} E_{n}^{T}\right) A_{n}^{-1} B_{n}\right) \\
& =4\left(2 x^{2}-6 x+3\right)\left(\operatorname{Tr}\left(A_{n}^{-1} E_{n} B_{n}\right)+\operatorname{Tr}\left(E_{n}^{T} A_{n}^{-1} B_{n}\right)\right) \\
& =8\left(2 x^{2}-6 x+3\right) \operatorname{Tr}\left(E_{n}^{T} A_{n}^{-1} B_{n}\right) .
\end{aligned}
$$


Since

$$
E_{n}^{T} A_{n}^{-1} B_{n}=\left[\begin{array}{ccccc}
a_{1} / 2 & a_{2} / 2 & \ldots & a_{n} / 2 & a_{0} \sigma_{0} / 2+\ldots \\
a_{0} & a_{1} & \ldots & a_{n-1} & a_{0} \sigma_{1}+\ldots \\
& \ddots & & & \vdots \\
& & \ddots & a_{1} & a_{0} \sigma_{n-1}+a_{1} \sigma_{n} \\
& 0 & & a_{0} & a_{0} \sigma_{n}
\end{array}\right]
$$

the trace reduces as follows:

$$
\begin{aligned}
& \operatorname{Tr}\left(A_{n}^{-1}\left[4\left(2 x^{2}-6 x+3\right) c_{i+j}\right]_{0 \leq i, j \leq n} A_{n}^{-1} B_{n}\right)=4\left(2 x^{2}-6 x+3\right)\left((2 n-1) a_{1}+2 a_{0} \sigma_{n}\right) \\
& =4(2 n-1)(x+4)\left(2 x^{2}-6 x+3\right) \\
& +8\left(2 x^{2}-6 x+3\right) \frac{K_{n}}{H_{n}} \text {. }
\end{aligned}
$$

Using the expansion (30), the final trace term (64) simplifies as follows:

$$
\operatorname{Tr}\left(A_{n}^{-1}\left[-27\left(2 x^{2}-6 x+3\right) c_{i+j-1}\right]_{0 \leq i, j \leq n} A_{n}^{-1} B_{n}\right)=-54 n\left(2 x^{2}-6 x+3\right) .
$$

Now we return to equation (55). In this equation, there is a term containing

$$
d_{x} \operatorname{Tr}\left(A_{n}^{-1} B_{n}\right) \text {. }
$$

We expanded the derivative above as the difference of two traces in (56). We multiplied (56) by $(x-3)(2 x-3)(4 x-3)$ and expressed the product

$$
\left.(x-3)(2 x-3)(4 x-3) d_{x} \operatorname{Tr}\left(A_{n}^{-1} B_{n}\right)\right)
$$

as the sum of four terms in (57), (58), (59), (60) minus the sum of four terms in (61), (62), (63), (64). These eight traces in turn were simplified as (65), (66), (67), (68), (69), (70), (71), (72). Computing the sum of (65), (66), (67), (68) minus the sum of (69), (70), (71), (72) we obtain

$$
\begin{aligned}
\left.H_{n}(x-3)(2 x-3)(4 x-3) d_{x} \operatorname{Tr}\left(A_{n}^{-1} B_{n}\right)\right)= & 2(4 n+5) N_{n}-2(4 n+1) M_{n} \\
& -27 K_{n}+(4 x-11)\left(2 x^{2}-6 x+3\right) H_{n} \\
& -2(4 n+3) \frac{K_{n}^{2}}{H_{n}} .
\end{aligned}
$$

Now we multiply this through by $2(4 n+3)$. The left hand side becomes the last term on the left hand side of equation (55). Substituting and simplifying, we obtain the intermediate identity

$$
\begin{array}{r}
(x-3)^{2}(2 x-3)^{2}(4 x-3)^{2} d_{x}^{2} H_{n}- \\
(x-3)(2 x-3)(4 x-3)\left(8(n-3) x^{2}-6(5 n-13) x-3\left(9 n^{2}+13 n+29\right)\right) d_{x} H_{n}- \\
2\left(64 n x^{4}-424 n x^{3}+2(475 n-6) x^{2}-3(283 n-15) x+3(91 n-6)\right) H_{n}- \\
2(4 n+3)\left(8 n x^{2}-6(5 n+1) x-3\left(9 n^{2}+13 n+17\right)\right) K_{n}+ \\
4(4 n+1)(4 n+3) M_{n}- \\
4(4 n+3)(4 n+5) N_{n}=0 .
\end{array}
$$

The EleCtronic Journal of COMBinatorics 15 (2008), \#R6 
Recall that equation (33) of Lemma 3 expresses $d_{x} H_{n}$ in terms of $H_{n}$ and $K_{n}$. Also, Lemma 4 gives a linear relationship between $H_{n}, K_{n}, M_{n}$ and $N_{n}$. Thus by using these two equations we can eliminate both the $d_{x} H_{n}$ and the $M_{n}$ term in the above formula:

$$
\begin{array}{r}
4(4 n+1)(x-1)(x-3)^{2}(2 x-3)^{2}(4 x-3)^{2} d_{x}^{2} H_{n}- \\
4(4 n+1)\left(64 n(n-1) x^{5}-96\left(3 n^{2}-8 n-2\right) x^{4}+12\left(36 n^{3}+163 n^{2}-65 n-8\right) x^{3}-\right. \\
4\left(459 n^{3}+1661 n^{2}+949 n+417\right) x^{2}+3\left(243 n^{4}+2106 n^{3}+5192 n^{2}+4291 n+1482\right) x \\
\left.-3\left(243 n^{4}+1674 n^{3}+3679 n^{2}+3140 n+1008\right)\right) H_{n}+ \\
8(4 n+1)(4 n+3)\left(16(n+2) x^{3}-4(17 n+31) x^{2}+6\left(9 n^{2}+48 n+53\right) x-\right. \\
\left.3\left(18 n^{2}+80 n+77\right)\right) K_{n}- \\
32(4 n+1)(4 n+3)(4 n+5)(x-1) N_{n}=0 .
\end{array}
$$

At this point we have enough information on the interrelationship between $H_{n}, K_{n}, N_{n}$ and $M_{n}$ that allows us to derive a number of evaluations of $H_{n}(x)$ for special values of $x$. These evaluations also turn out to be essential for the final step of the proof of Theorem 1 in Section 4.

\section{Product form evaluations of $H_{n}(x)$ at special $x$}

Our calculations for the special values rely on identities we have proved for $H_{n}, K_{n}, N_{n}$ and $M_{n}$ as well as a Dodgson-like determinant identity which is useful. This identity is as follows:

Proposition 1 Suppose the determinants $H_{n}, K_{n}, M_{n}$, and $N_{n}$ are defined as in (13), (21), (34), and (35) respectively. Then

$$
H_{n-1} H_{n+1}=H_{n} N_{n}-H_{n} M_{n}-K_{n}^{2}
$$

Proof This identity can be proved using techniques similar to those in [9]. We first prove a general determinant expansion result, and then specialize to Hankel determinants to obtain (75). Consider two matrices

$$
\begin{aligned}
R_{n} & =\left[r_{i, j}\right]_{0 \leq i, j \leq n} \\
X_{n+1} & =\left[x_{i, j}\right]_{0 \leq i, j \leq n+1}
\end{aligned}
$$

where ultimately we will set for all $i, j$

$$
r_{i, j}=x_{i, j}=a_{i+j}(x)
$$


Consider the sum

$$
\sum_{k=0}^{n+1}(-1)^{n+1-k}\left(\operatorname { d e t } \left[\left\{\begin{array}{ll}
r_{i, j} & \text { if } i \neq n \\
x_{k, j+1} & \text { if } i=n
\end{array}\right]_{0 \leq i, j \leq n} \operatorname{det}\left[\left\{\begin{array}{ll}
x_{i, j} & \text { if } i<k \\
x_{i+1, j} & \text { if } i \geq k
\end{array}\right]_{0 \leq i, j \leq n}\right)\right.\right.
$$

as a function of the matrix $X=X_{n+1}$. It is not difficult to prove that if two adjacent rows of $X$ are switched then the sum (76) changes sign. Since the set of all permutations of the rows is generated by flips of adjacent rows, it follows that (76) is alternating.

In addition (76) is linear in each row of $X$. Since (76) is both alternating and multilinear, it is equal to a multiple (depending on $R_{n}$ ) of $\operatorname{det}\left(X_{n+1}\right)$. To determine the multiple we set $X$ to the matrix:

$$
X_{n+1}=\left[\begin{array}{ccccc}
1 & 0 & \ldots & & 0 \\
0 & r_{0,0} & r_{0,1} & \ldots & r_{0, n} \\
0 & r_{1,0} & r_{1,1} & \ldots & r_{1, n} \\
\vdots & & & & \\
0 & r_{n, 0} & r_{n, 1} & \ldots & r_{n, n}
\end{array}\right]
$$

In this case

$$
\operatorname{det}\left(X_{n+1}\right)=\operatorname{det}\left(R_{n}\right) .
$$

In the sum (76) only the term corresponding to $i=n+1$ survives and this term itself evaluates to

$$
\operatorname{det}\left(R_{n}\right) \operatorname{det}\left(R_{n-1}\right) \text {. }
$$

Therefore for all $R$ and $X$, the sum (76) evaluates to $\operatorname{det}\left(R_{n-1}\right) \operatorname{det}\left(X_{n+1}\right)$. When $R$ and $X$ are Hankel, only the last three terms of in the sum (76) survive. In particular, if we set

$$
r_{i, j}=x_{i, j}=a_{i+j}(x)
$$

we obtain identity (75).

\subsection{The evaluation of $H_{n}(3)$}

We use the identities involving $H_{n}, K_{n}, M_{n}$, and $N_{n}$ that we already have, along with (75). At the point $x=3$ the left hand side of (75) evaluates to $H_{n-1}(3) H_{n+1}(3)$. The right hand side of (75) is more difficult to evaluate; we have to use the identities from the previous section. At $x=3$, equation (33) simplifies to

$$
2(4 n+3) K_{n}(3)=3\left(9 n^{2}+19 n+14\right) H_{n}(3) .
$$

Therefore

$$
K_{n}(3)=\frac{3\left(9 n^{2}+19 n+14\right)}{2(4 n+3)} H_{n}(3)
$$


Now we can use this together with equation (74) to calculate $N_{n}$ at $x=3$

$$
N_{n}(3)=\frac{3\left(243 n^{4}+1512 n^{3}+3605 n^{2}+4144 n+1920\right)}{8(4 n+3)(4 n+5)} H_{n}(3) .
$$

Finally we use the linear relationship of Lemma 4 to calculate $M_{n}$ at $x=3$ :

$$
M_{n}(3)=-\frac{3 n\left(243 n^{3}+540 n^{2}+559 n+58\right)}{8(4 n+1)(4 n+3)} H_{n}(3)
$$

We can now write the right hand side of (75) as

$$
\frac{9(3 n+4)(3 n+5)(6 n-1)(6 n+1)}{4(4 n+1)(4 n+3)^{2}(4 n+5)} H_{n}(3)^{2} .
$$

Letting $H_{n}=H_{n}(3)$, we have

$$
H_{n-1} H_{n+1}=\frac{9(3 n+4)(3 n+5)(6 n-1)(6 n+1)}{4(4 n+1)(4 n+3)^{2}(4 n+5)} H_{n}^{2} .
$$

Writing (81) as a recursion for $\frac{H_{n+1}}{H_{n}}$, we find that

$$
\frac{H_{n+1}}{H_{n}}=\frac{H_{1}}{H_{0}} \prod_{i=1}^{n} \frac{9(3 i+4)(3 i+5)(6 i-1)(6 i+1)}{4(4 i+1)(4 i+3)^{2}(4 i+5)} .
$$

Thus

$$
H_{n}=H_{0}\left(\frac{H_{1}}{H_{0}}\right)^{n} \prod_{j=1}^{n-1} \prod_{i=1}^{n-j} \frac{9(3 i+4)(3 i+5)(6 i-1)(6 i+1)}{4(4 i+1)(4 i+3)^{2}(4 i+5)} .
$$

We compute directly that $H_{0}=1$ and $H_{1}=-1$. Rearranging,

$$
H_{n}=(-1)^{n} \prod_{j=1}^{n-1} \prod_{i=1}^{n-j} \frac{9(3 i+4)(3 i+5)(6 i-1)(6 i+1)}{4(4 i+1)(4 i+3)^{2}(4 i+5)} .
$$

This double product expression for $H_{n}$ can be rewritten in terms of factorials. We record this as a corollary.

Corollary 1 Suppose $a_{k}(x)$ is defined as in (7) and $H_{n}(x)=\operatorname{det}\left[a_{i+j}(x)\right]_{0 \leq i, j \leq n}$. Then

$$
H_{n}(3)=(-1)^{n} \prod_{i=1}^{n} \frac{(6 i-3) !(3 i+2) !(2 i-1) !}{(4 i-1) !(4 i+1) !(3 i-2) !} .
$$




\subsection{The evaluation of $H_{n}\left(\frac{3}{2}\right)$}

For $x=\frac{3}{2}$, the left hand side of identity (33) of Lemma 3 vanishes, and we obtain

$$
K_{n}\left(\frac{3}{2}\right)=\frac{3\left(9 n^{2}+22 n+11\right)}{2(4 n+3)} H_{n}\left(\frac{3}{2}\right) .
$$

Next, we evaluate (74) at $x=\frac{3}{2}$ using this expression for $K_{n}$. The second derivative terms drops out and we obtain

$$
N_{n}\left(\frac{3}{2}\right)=\frac{3\left(243 n^{4}+1674 n^{3}+4031 n^{2}+3934 n+1290\right)}{8(4 n+3)(4 n+5)} H_{n}\left(\frac{3}{2}\right) .
$$

Finally, using these expressions for $K_{n}\left(\frac{3}{2}\right)$ and $N_{n}\left(\frac{3}{2}\right)$ in the linear identity (36) of Lemma 4 at $x=\frac{3}{2}$, we obtain

$$
M_{n}\left(\frac{3}{2}\right)=\frac{-3 n\left(243 n^{3}+702 n^{2}+547 n+118\right)}{8(4 n+1)(4 n+3)} H_{n}\left(\frac{3}{2}\right) .
$$

Substituting these in (75) with $H_{n}=H_{n}\left(\frac{3}{2}\right)$, we obtain

$$
H_{n-1} H_{n+1}=\frac{9(3 n+2)(3 n+4)(6 n+1)(6 n+5)}{4(4 n+1)(4 n+3)^{2}(4 n+5)} H_{n}^{2} .
$$

This can be written as a recursion for $\frac{H_{n}}{H_{n-1}}$ giving

$$
H_{n}=H_{n-1} \cdot \frac{H_{1}}{H_{0}} \prod_{i=1}^{n-1} \frac{9(3 i+2)(3 i+4)(6 i+1)(6 i+5)}{4(4 i+1)(4 i+3)^{2}(4 i+5)} .
$$

Iterating (88), and using the fact that $H_{0}\left(\frac{3}{2}\right)=1$ and $H_{1}\left(\frac{3}{2}\right)=2$, we obtain the product formula

$$
H_{n}\left(\frac{3}{2}\right)=2^{n} \cdot \prod_{j=1}^{n-1} \prod_{i=1}^{n-j} \frac{9(3 i+2)(3 i+4)(6 i+1)(6 i+5)}{4(4 i+1)(4 i+3)^{2}(4 i+5)} .
$$

The expression (89) can equivalently be written in terms of factorials as in (90).

Corollary 2 Suppose $a_{k}(x)$ is defined as in (7) and $H_{n}(x)=\operatorname{det}\left[a_{i+j}(x)\right]_{0 \leq i, j \leq n}$. Then

$$
H_{n}\left(\frac{3}{2}\right)=\prod_{i=1}^{n} \frac{(2 i-1) !(6 i) !(3 i+1)}{2(4 i-1) !(4 i+1) !} .
$$




\subsection{The evaluation of $H_{n}\left(\frac{3}{4}\right)$}

We can mimic the above steps for the evaluation of $H_{n}$ at $x=\frac{3}{4}$ as well. Evaluating the identities (33) of Lemma 3, (74), and (36) of Lemma 4 at $x=\frac{3}{4}$, we obtain respectively,

$$
\begin{aligned}
& K_{n}\left(\frac{3}{4}\right)=\frac{3\left(18 n^{2}+38 n+19\right)}{4(4 n+3)} H_{n}\left(\frac{3}{4}\right) \\
& N_{n}\left(\frac{3}{4}\right)=\frac{3\left(486 n^{4}+3024 n^{3}+6724 n^{2}+6290 n+2085\right)}{16(4 n+3)(4 n+5)} H_{n}\left(\frac{3}{4}\right) \\
& M_{n}\left(\frac{3}{4}\right)=\frac{-3 n\left(243 n^{3}+540 n^{2}+316 n+31\right)}{8(4 n+1)(4 n+3)} H_{n}\left(\frac{3}{4}\right) .
\end{aligned}
$$

Substituting these in (75) with $H_{n}=H_{n}\left(\frac{3}{4}\right)$, we obtain the recursion

$$
H_{n-1} H_{n+1}=\frac{9(3 n+1)(3 n+2)(6 n+5)(6 n+7)}{4(4 n+1)(4 n+3)^{2}(4 n+5)} H_{n}^{2} .
$$

Using the values $H_{0}\left(\frac{3}{4}\right)=1$ and $H_{1}\left(\frac{3}{2}\right)=\frac{7}{2}$, and calculating as in the derivation of (89), we obtain

$$
H_{n}\left(\frac{3}{4}\right)=\left(\frac{7}{2}\right)^{n} \prod_{j=1}^{n-1} \prod_{i=1}^{n-j} \frac{9(3 i+1)(3 i+2)(6 i+5)(6 i+7)}{4(4 i+1)(4 i+3)^{2}(4 i+5)} .
$$

The expression (94) can be written in terms of factorials as given in (95).

Corollary 3 Suppose $a_{k}(x)$ is defined as in (7) and $H_{n}(x)=\operatorname{det}\left[a_{i+j}(x)\right]_{0 \leq i, j \leq n}$. Then

$$
H_{n}\left(\frac{3}{4}\right)=\prod_{i=1}^{n} \frac{(2 i-1) !(6 i+1) !}{2(4 i-1) !(4 i+1) !} .
$$

\section{A third identity and the differential equation for $H_{n}$}

We now continue with the proof of Theorem 1. Using the expression for $d_{x}^{2} H_{n}$ in terms of $H_{n}, K_{n}$ and $N_{n}$ from (74), and the expression for $d_{x} H_{n}$ in terms of $H_{n}$ and $K_{n}$ from (33), we have

$$
\begin{aligned}
& (x-3)(2 x-3)^{2}(4 x-3)^{2}\left((x-1)(x-3) d_{x}^{2} H_{n}\right. \\
& \left.+(2(n+2)(x-3)+3) d_{x} H_{n}-3 n(n+1) H_{n}\right) \\
& =8(4 n+3)(4 n+5)(x-1) N_{n} \\
& -4(4 n+3)\left(-27 n^{2}-93 n-75+\left(27 n^{2}+81 n+60\right) x+(8 n+10) x^{2}\right) K_{n} \\
& -\left(729 n^{4}+3564 n^{3}+6015 n^{2}+4236 n+1080\right. \\
& -\left(729 n^{4}+2916 n^{3}+3777 n^{2}+1722 n+180\right) x \\
& \left.-\left(432 n^{3}+1492 n^{2}+1676 n+600\right) x^{2}\right) H_{n} .
\end{aligned}
$$


To prove Theorem 1, we show that the expression on the right hand side of (96) is identically zero. This is the consequence of our third identity.

Write the right hand side of (96) in the form

$$
-\left(p_{n} N_{n}+q_{n} K_{n}+r_{n} H_{n}\right)
$$

where $p_{n}=p_{n}(x), q_{n}=q_{n}(x)$, and $r_{n}=r_{n}(x)$ are the negatives of the polynomials multiplying $N_{n}, K_{n}$, and $H_{n}$ on the right hand side of (96), respectively. That is,

$$
\begin{gathered}
p_{n}(x)=-8(4 n+3)(4 n+5)(x-1) \\
q_{n}(x)=4(4 n+3)\left(-27 n^{2}-93 n-75+\left(27 n^{2}+81 n+60\right) x+(8 n+10) x^{2}\right) \\
r_{n}(x)=729 n^{4}+3564 n^{3}+6015 n^{2}+4236 n+1080 \\
\quad-\left(729 n^{4}+2916 n^{3}+3777 n^{2}+1722 n+180\right) x \\
\quad-\left(432 n^{3}+1492 n^{2}+1676 n+600\right) x^{2} .
\end{gathered}
$$

Note that $p_{n}$ is linear, while $q_{n}$ and $r_{n}$ are quadratic polynomials in $x$.

The three matrices in (13), (21), (35) that define $H_{n}, K_{n}$, and $N_{n}$ respectively, differ only in their last column. Therefore the expression

$$
p_{n} N_{n}+q_{n} K_{n}+r_{n} H_{n}
$$

is the determinant of the $(n+1) \times(n+1)$ matrix whose

i) first $n$ columns are the columns of the matrix $A_{n}$ in (13),

ii) whose last column is the linear combination

$$
p_{n} v_{n+2}+q_{n} v_{n+1}+r_{n} v_{n}
$$

where for $j \geq 0$

$$
v_{j}=\left[\begin{array}{c}
a_{j} \\
a_{j+1} \\
\vdots \\
a_{j+n}
\end{array}\right] .
$$

To show that the determinant (99) is zero, it suffices to show that the last column (100) of this matrix is a linear combination of the first $n$ columns $v_{0}, v_{1}, \ldots, v_{n-1}$, i.e. there are weights $w_{n, 0}, w_{n, 1}, \ldots, w_{n, n+2}$ with

$$
\begin{aligned}
w_{n, n+2} & =p_{n} \\
w_{n, n+1} & =q_{n} \\
w_{n, n} & =r_{n}
\end{aligned}
$$


such that

$$
\sum_{j=0}^{n+2} w_{n, j} a_{i+j}=0
$$

for $i=0,1, \ldots, n$. This is the third identity we need.

After some experimentation, bolstered by checks in Mathematica for values up to $n=30$, we found that the weights are likely given by

$$
w_{n, j}(x)=\sum_{i=0}^{\min \left(-j+n+1,\left\lfloor\frac{n}{2}\right\rfloor+1\right)} A_{n, i, j}(x)+\sum_{i=0}^{\min \left(-j+n+2,\left\lfloor\frac{n}{2}\right\rfloor+1\right)} B_{n, i, j}(x)
$$

where

$$
\begin{aligned}
& A_{n, i, j}(x)=u_{n, i}\left(\alpha_{n, i, j}+\beta_{n, i, j} x+\gamma_{n, i, j} x^{2}\right) \\
& B_{n, i, j}(x)=v_{n, i}\left(\delta_{n, i, j}+\epsilon_{n, i, j} x+\theta_{n, i, j} x^{2}\right)
\end{aligned}
$$

and

$$
\begin{aligned}
& u_{n, i}=\frac{n u_{n, i}}{d u_{n, i}} \text { with } \\
& n u_{n, i}=3 \cdot 2^{i+1}(4 n+3)(4 n+5)\left(\begin{array}{c}
n \\
2 i
\end{array}\right)\left(\begin{array}{l}
j+2(n-i+1)+1 \\
2(n-i-j+1)+1
\end{array}\right) . \\
& (2 n-i+2) !(4 n-2 i+5) ! !(6 n+7) ! ! \\
& d u_{n, i}=(2 i+1)(3 j+1)(3 j+2)(2 i-j-2 n-3)(2 i-j-2 n-2) \cdot \\
& (2 n+1) !(4 n+5) ! !(6 n-4 i+7) ! !, \\
& \alpha_{n, i, j}=3(9 j(j+1)+2)\left(12 i^{2}+6(3 j-4 n-5) i+6(n+1)(2 n+3)-j(18 n+19)\right) \text {, } \\
& \beta_{n, i, j}=-3(3 j+2)\left(24 i^{3}+8(12 j-9 n-8) i^{2}\right. \\
& +2\left(36 n^{2}-96 j n+64 n+9 j(5 j-11)+23\right) i \\
& \left.-2(n+1)(2 n+3)(6 n+1)-j^{2}(90 n+89)+3 j\left(32 n^{2}+66 n+33\right)\right), \\
& \gamma_{n, i, j}=2(2 i+2 j-2 n-3)(i+j-n-1)\left(36 i^{2}+6(9 j-12 n-5) i\right. \\
& +6 n(6 n+5)-3 j(18 n+13)+2) \text {, } \\
& v_{n, i}=\frac{n v_{n, i}}{d v_{n, i}} \text { with } \\
& n v_{n, i}=3 \cdot 2^{i+3}(2 n-2 i-2 j+5)(4 n+3)(4 n+5)\left(\begin{array}{c}
n+1 \\
2 i
\end{array}\right)\left(\begin{array}{c}
j+2(n-i+2)+1 \\
2(n-i-j+2)+1
\end{array}\right) . \\
& (2 n-i+3) !(4 n-2 i+5) ! !(6 n+7) ! ! \\
& d v_{n, i}=(3 j+1)(3 j+2)(2 i-j-2 n-5)(2 i-j-2 n-4)(2 i-j-2 n-3) . \\
& (2 n+2) !(4 n+5) ! !(6 n-4 i+9) ! ! \\
& \delta_{n, i, j}=3(9 j(j+1)+2)\left(6 i^{2}+3(3 j-4 n-7) i+3(n+2)(2 n+3)-j(9 n+14)\right), \\
& \epsilon_{n, i, j}=-3(3 j+2)\left(12 i^{3}+(48 j-36 n-50) i^{2}\right.
\end{aligned}
$$




$$
\begin{gathered}
+\left(45 j^{2}-3(32 n+49) j+4(n+1)(9 n+16)\right) i \\
\left.-2(n+2)(2 n+3)(3 n+2)-j^{2}(45 n+67)+3 j(n(16 n+49)+37)\right), \\
\theta_{n, i, j}=2(2 i+2 j-2 n-3)(i+j-n-2)\left(18 n^{2}-9(4 i+3 j) n\right. \\
+33 n-33 j+3 i(6 i+9 j-11)+13) .
\end{gathered}
$$

The proof of formula (102), and consequently the proof of Theorem 1, is therefore reduced to a somewhat involved system of binomial identities involving summations over multiple indices. Our first reaction was that a pen-and-paper proof was infeasible. The explicit form of the conjectured weights gave us a slim hope that automated binomial identity provers might work. However, we did not see how to do this. So our entire approach to the evaluation of the determinants $H_{n}$ seemed to founder on these identities. Our efforts seemed to indicate that these identities may well be beyond the standard formulations and computational resources required for automated binomial identity provers, and even if such a proof is possible, the insight such it would offer would be minimal.

We were able to find an alternative approach, which avoids the explicit form of the weights, and which has the advantage of generalization to other cases. For the proof, all we need to do is to show the existence of weights satisfying (102) and (101). This becomes a manageable problem which relies on certain properties of the generating function

$$
f=f(x, y)=\sum_{k \geq 0} a_{k}(x) y^{k}
$$

of the $a_{k}$.

Lemma 5 Suppose $a_{k}(x)$ is as defined in (7) and $f$ is as in (104). Then

$$
f(x, y)=\frac{4 x+2 t-6}{(x-3)(4 x-3) y(2 t-3)-(x-1)(27 y-4)}
$$

where

$$
t=\sum_{k \geq 0} \frac{(3 k) !}{(2 k+1) ! k !} y^{k}=1+y+3 y^{2}+12 y^{3}+\cdots
$$

satisfies $t^{3} y=t-1$.

The proof of the lemma can be found in Appendix I.

The proof of the existence of weights satisfying (102) and (101) takes several steps. Suppose $t$ is as in Lemma 5 and define

$$
\eta=2 t-3=-1+2 y+6 y^{2}+\cdots
$$

Then

$$
f(x, y)=\frac{4 x-3+\eta}{(x-3)(4 x-3) y \eta-(x-1)(27 y-4)} .
$$


The first step is to note that we can find a nontrivial polynomial $Q_{0}=Q_{0}(y)$ of degree $n+1$ such that in the series expansion

$$
\eta Q_{0}=b_{0}+b_{1} y+b_{2} y^{2}+\cdots
$$

we have

$$
b_{n+2}=b_{n+3}=\cdots=b_{2 n+2}=0 .
$$

This is possible because there are $n+2$ coefficients available in $Q_{0}$ and only $n+1$ homogeneous linear equations imposed by (107) that these coefficients are required to satisfy. Thus we can write

$$
\eta Q_{0}=Q_{1}+y^{2 n+3} \Psi_{0}
$$

where $Q_{1}=Q_{1}(y)$ is a polynomial of degree $n+1$, and $\Psi_{0}=\Psi_{0}(y)$ is a power series in $y$. In the next step, put

$$
Q_{2}=(x-3)(4 x-3) y Q_{1}-(x-1)(27 y-4) Q_{0} .
$$

Then $Q_{2}=Q_{2}(x, y)$ is a polynomial in $x$ and $y$ of $y$-degree $n+2$. It is important to note that all three of the polynomials $Q_{0}, Q_{1}, Q_{2}$ so defined are nontrivial. We claim that the coefficients of $Q_{2}$ are the weights we want. In other words, the coefficients of the terms $y^{n+2}$ through $y^{2 n+2}$ in $f Q_{2}$ vanish. Our aim now is to use the special form of the generating function $f$ in (105) to show this.

Writing (105) in the form

$$
f(x, y)((x-3)(4 x-3) y \eta-(x-1)(27 y-4))=4 x-3+\eta
$$

and multiplying through by $Q_{0}$, we get

$f(x, y)\left((x-3)(4 x-3) y\left(Q_{1}+y^{2 n+3} \Psi_{0}\right)-(x-1)(27 y-4) Q_{0}\right)=(4 x-3) Q_{0}+Q_{1}+y^{2 n+3} \Psi_{0}$

or

$$
f(x, y)\left(Q_{2}+(x-3)(4 x-3) y^{2 n+4} \Psi_{0}\right)=(4 x-3) Q_{0}+Q_{1}+y^{2 n+3} \Psi_{0}
$$

and therefore

$$
f(x, y) Q_{2}=(4 x-3) Q_{0}+Q_{1}+y^{2 n+3}\left(\Psi_{0}+f(x, y)(x-3)(4 x-3) y \Psi_{0}\right)
$$

which means that

$$
f Q_{2}=(4 x-3) Q_{0}+Q_{1}+y^{2 n+3} \Psi_{1}
$$

where

$$
(4 x-3) Q_{0}+Q_{1}
$$

is a polynomial in $x$ and $y$ of $y$-degree $n+1$ and $\Psi_{1}=\Psi(y)$ is a power series in $y$. 
This last statement (110) is equivalent to the statement that

$$
\sum_{j=0}^{n+2} \mathcal{C}_{n+2-j}\left(Q_{2}\right) a_{i+j}=0
$$

for $i=0,1, \ldots, n$, where by $\mathcal{C}_{k}(\Psi)$ we denote the coefficient of the term $y^{k}$ in a power series $\Psi$. Thus (102) holds with

$$
w_{n, j}=\mathcal{C}_{n+2-j}\left(Q_{2}\right)
$$

for $j=0,1, \ldots, n+2$.

In particular, taking determinants, we have

$$
\mathcal{C}_{0}\left(Q_{2}\right) N_{n}+\mathcal{C}_{1}\left(Q_{2}\right) K_{n}+\mathcal{C}_{2}\left(Q_{2}\right) H_{n}=0
$$

This identity is not trivial. Otherwise, we would have a nontrivial linear relationship among the $n+1$ columns $v_{0}, v_{1}, \ldots, v_{n}$ of $H_{n}$. But we have already shown that the special values $H_{n}(3), H_{n}\left(\frac{3}{2}\right), H_{n}\left(\frac{3}{4}\right)$ evaluated in (83), (90), (95) are nonzero, and therefore $H_{n}$ does not identically vanish.

Now we need to verify the three special values in (101). Since equation (111) is valid when multiplied through by an arbitrary constant, in terms of the coefficients $\mathcal{C}_{k}\left(Q_{2}\right)$, we need to show that for some nonzero constant $\alpha$,

$$
\begin{aligned}
& \mathcal{C}_{0}\left(Q_{2}\right)=\alpha p_{n} \\
& \mathcal{C}_{1}\left(Q_{2}\right)=\alpha q_{n} \\
& \mathcal{C}_{2}\left(Q_{2}\right)=\alpha r_{n}
\end{aligned}
$$

where $p_{n}, q_{n}, r_{n}$ are as defined in (98).

First we rewrite $(112)$ in terms of the three coefficients $\mathcal{C}_{0}\left(Q_{0}\right), \mathcal{C}_{1}\left(Q_{0}\right), \mathcal{C}_{2}\left(Q_{0}\right)$ which are pure constants, independent on $x$ and $y$. First, comparing coefficients in (108) and (109), we obtain the five identities in (114).

$$
\begin{aligned}
& \mathcal{C}_{0}\left(Q_{1}\right)=-\mathcal{C}_{0}\left(Q_{0}\right) \\
& \mathcal{C}_{1}\left(Q_{1}\right)=2 \mathcal{C}_{0}\left(Q_{0}\right)-\mathcal{C}_{1}\left(Q_{0}\right) \\
& \mathcal{C}_{0}\left(Q_{2}\right)=4(x-1) \mathcal{C}_{0}\left(Q_{0}\right) \\
& \mathcal{C}_{1}\left(Q_{2}\right)=(x-3)(4 x-3) \mathcal{C}_{0}\left(Q_{1}\right)-27(x-1) \mathcal{C}_{0}\left(Q_{0}\right)+4(x-1) \mathcal{C}_{1}\left(Q_{0}\right) \\
& \left.\mathcal{C}_{2}\left(Q_{2}\right)=(x-3)(4 x-3) \mathcal{C}_{1}\left(Q_{1}\right)-27(x-1)\right) \mathcal{C}_{1}\left(Q_{0}\right)+4(x-1) \mathcal{C}_{2}\left(Q_{0}\right)
\end{aligned}
$$

Therefore

$$
\begin{aligned}
& \mathcal{C}_{0}\left(Q_{2}\right)=4(x-1) \mathcal{C}_{0}\left(Q_{0}\right) \\
& \mathcal{C}_{1}\left(Q_{2}\right)=-2\left(2 x^{2}+6 x-9\right) \mathcal{C}_{0}\left(Q_{0}\right)+4(x-1) \mathcal{C}_{1}\left(Q_{0}\right) \\
& \mathcal{C}_{2}\left(Q_{2}\right)=2(x-3)(4 x-3) \mathcal{C}_{0}\left(Q_{0}\right)-2\left(2 x^{2}+6 x-9\right) \mathcal{C}_{1}\left(Q_{0}\right)+4(x-1) \mathcal{C}_{2}\left(Q_{0}\right)
\end{aligned}
$$


and (112) becomes

$$
\begin{aligned}
& 4(x-1) \mathcal{C}_{0}\left(Q_{0}\right) N_{n}+ \\
& \left(-2\left(2 x^{2}+6 x-9\right) \mathcal{C}_{0}\left(Q_{0}\right)+4(x-1) \mathcal{C}_{1}\left(Q_{0}\right)\right) K_{n}+ \\
& \left(2(x-3)(4 x-3) \mathcal{C}_{0}\left(Q_{0}\right)-2\left(2 x^{2}+6 x-9\right) \mathcal{C}_{1}\left(Q_{0}\right)+4(x-1) \mathcal{C}_{2}\left(Q_{0}\right)\right) H_{n}=0 .
\end{aligned}
$$

We have explicit linear relationships between the three determinants $N_{n}, K_{n}, H_{n}$ at the points $x=3, \frac{3}{2}, \frac{3}{4}$. For $x=3$, we use the expressions in terms of $H_{n}(3)$ for $N_{n}(3)$ from (79) and for $K_{n}(3)$ from (79) in (116) to obtain the linear equation

$$
\begin{aligned}
& 8(3+4 n)(5+4 n) \mathcal{C}_{2}\left(Q_{0}\right)+ \\
& 6(5+4 n)\left(1+2 n+18 n^{2}\right) \mathcal{C}_{1}\left(Q_{0}\right)+ \\
& \quad 3\left(30+67 n+338 n^{2}+540 n^{3}+243 n^{4}\right) \mathcal{C}_{0}\left(Q_{0}\right)=0 .
\end{aligned}
$$

For $x=\frac{3}{2}$, we use the expressions in terms of $H_{n}\left(\frac{3}{2}\right)$ for $N_{n}\left(\frac{3}{2}\right)$ from $(85)$ and for $K_{n}\left(\frac{3}{2}\right)$ from (84) in (116) to obtain

$$
\begin{aligned}
& 8(3+4 n)(5+4 n) \mathcal{C}_{2}\left(Q_{0}\right)+ \\
& 12(5+4 n)\left(2+10 n+9 n^{2}\right) \mathcal{C}_{1}\left(Q_{0}\right)+ \\
& \quad 3\left(120+778 n+1445 n^{2}+1026 n^{3}+243 n^{4}\right) \mathcal{C}_{0}\left(Q_{0}\right)=0 .
\end{aligned}
$$

Finally for $x=\frac{3}{4}$, we use (92) and (91) in (116) to obtain

$$
\begin{aligned}
& 8(3+4 n)(5+4 n) \mathcal{C}_{2}\left(Q_{0}\right)+ \\
& 12(5+4 n)\left(-4+n+9 n^{2}\right) \mathcal{C}_{1}\left(Q_{0}\right)+ \\
& \quad 3\left(-240-446 n+95 n^{2}+540 n^{3}+243 n^{4}\right) \mathcal{C}_{0}\left(Q_{0}\right)=0 .
\end{aligned}
$$

Using (any two of) these equations we obtain the parametric solutions

$$
\begin{aligned}
& \mathcal{C}_{2}\left(Q_{0}\right)=3\left(50 n+343 n^{2}+540 n^{3}+243 n^{4}\right) \alpha \\
& \mathcal{C}_{1}\left(Q_{0}\right)=-12(1+n)\left(30+67 n+36 n^{2}\right) \alpha \\
& \mathcal{C}_{0}\left(Q_{0}\right)=8\left(15+32 n+16 n^{2}\right) \alpha .
\end{aligned}
$$

Going back to the system (115), we compute that

$$
\begin{aligned}
\mathcal{C}_{0}\left(Q_{2}\right)= & 32(4 n+3)(4 n+5)(x-1) \alpha \\
\mathcal{C}_{1}\left(Q_{2}\right)= & -16(4 n+3)\left(-75-93 n-27 n^{2}+60 x+81 n x+27 n^{2} x+10 x^{2}+8 n x^{2}\right) \alpha \\
\mathcal{C}_{2}\left(Q_{2}\right)= & 4\left(-1080-4236 n-6015 n^{2}-3564 n^{3}-729 n^{4}+180 x+1722 n x+3777 n^{2} x\right. \\
& \left.\quad+2916 n^{3} x+729 n^{4} x+600 x^{2}+1676 n x^{2}+1492 n^{2} x^{2}+432 n^{3} x^{2}\right) \alpha .
\end{aligned}
$$

Taking $\alpha=-\frac{1}{4}$, these are exactly the weights $w_{n, n+2}=p_{n}, w_{n, n+1}=q_{n}, w_{n, n}=r_{n}$ as claimed in (101). This finishes the proof of Theorem 1. 


\subsection{The differential equation solution for the $(3,1)$-case}

In the previous section we derived the differential equation (14) satisfied by

$$
H_{n}(x)=\operatorname{det}\left[\begin{array}{ccccc}
a_{0} & a_{1} & a_{2} & \ldots & a_{n} \\
a_{1} & a_{2} & a_{3} & \ldots & a_{n+1} \\
\vdots & & & & \vdots \\
a_{n} & a_{n+1} & \ldots & & a_{2 n}
\end{array}\right]
$$

where

$$
a_{k}=a_{k}^{(3,1)}(x)=\sum_{m=0}^{k}\left(\begin{array}{c}
3 k+1-m \\
k-m
\end{array}\right) x^{m} .
$$

We obtain a power series solution to (14) of the form

$$
y(x)=\sum_{i=0}^{\infty} b_{i}(x-3)^{i}
$$

where the $b_{i}$ satisfy

$$
b_{i+1}=\frac{(3 n+i+3)(n-i)}{(k+1)(2 k+3)} b_{i}
$$

with $b_{0}=1$. This allows us to prove that

$$
H_{n}(x)=C_{n} \sum_{i=0}^{n} \frac{n !(3 n+i+2) ! 2^{i}(x-3)^{i}}{(3 n+2) !(n-i) !(2 i+1) !}
$$

where $C_{n}$ is the constant of integration that depends only on $n$ but not on $x$. We have already calculated $C_{n}=H_{n}(3)$ in (83) of Corollary 1 . We record the final determinant evaluation result of the $(3,1)$-case as a Theorem:

Theorem 2 Suppose $a_{k}(x)$ is defined as in (7) and $H_{n}(x)=\operatorname{det}\left[a_{i+j}(x)\right]_{0 \leq i, j \leq n}$. Then

$$
H_{n}(x)=(-1)^{n} \prod_{i=1}^{n} \frac{(6 i-3) !(3 i+2) !(2 i-1) !}{(4 i-1) !(4 i+1) !(3 i-2) !} \sum_{i=0}^{n} \frac{n !(3 n+i+2) ! 2^{i}(x-3)^{i}}{(3 n+2) !(n-i) !(2 i+1) !} .
$$

In particular, evaluating (123) at $x=0$ and at $x=1$ we obtain

\section{Corollary 4}

$$
\begin{aligned}
& \operatorname{det}\left[\left(\begin{array}{c}
3(i+j)+1 \\
i+j
\end{array}\right)\right]_{0 \leq i, j \leq n}= \\
& (-1)^{n} \prod_{i=1}^{n} \frac{(6 i-3) !(3 i+2) !(2 i-1) !}{(4 i-1) !(4 i+1) !(3 i-2) !} \sum_{i=0}^{n} \frac{n !(3 n+i+2) !(-6)^{i}}{(3 n+2) !(n-i) !(2 i+1) !} \\
& \operatorname{det}\left[\left(\begin{array}{c}
3(i+j)+2 \\
i+j
\end{array}\right)\right]_{0 \leq i, j \leq n}= \\
& (-1)^{n} \prod_{i=1}^{n} \frac{(6 i-3) !(3 i+2) !(2 i-1) !}{(4 i-1) !(4 i+1) !(3 i-2) !} \sum_{i=0}^{n} \frac{n !(3 n+i+2) !(-4)^{i}}{(3 n+2) !(n-i) !(2 i+1) !}
\end{aligned}
$$


The formula on the right-hand side of (126) does indeed simplify to the product formula (3) by means of the Chu-Vandermonde summation formula.

\subsection{A recursion for $H_{n}^{(3,1)}(x)$}

We now remark on a property of the polynomials $H_{n}(x)$ for the $(3,1)$-case, which does not enter into the evaluation of the determinant, but which nevertheless is of interest.

Theorem 3 With $H_{0}=1, H_{1}(x)=-2 x+5$, the $H_{n}(x)$ satisfy a three-term polynomial recursion

$$
p_{n}(x) H_{n}(x)+q_{n}(x) H_{n-1}(x)+H_{n-2}(x)=0
$$

for $n \geq 2$ where

$$
\begin{aligned}
p_{n}(x)= & \frac{4(4 n-3) !^{2}(4 n-1) !^{2}}{9(3 n-2)^{2}(3 n-1)^{2}(2 n-1) !^{2}(6 n-5) !^{2}}(x-1)^{2} \\
q_{n}(x)= & \frac{4(n-1)(4 n-5) !(4 n-1) !}{3(3 n-2)(3 n-1)(4 n+1)(2 n-1) !(6 n-5) !}\left(8(4 n-3)(4 n+1) x^{3}\right. \\
& \left.\quad-36(4 n-3)(4 n+1) x^{2}+6\left(126 n^{2}-63 n-23\right) x-3\left(108 n^{2}-54 n-19\right)\right)
\end{aligned}
$$

Proof The theorem follows from the explicit form of the $H_{n}(x)$ given in Theorem 2 . We omit the details.

The following property of the $H_{n}$ for the $(3,1)$-case is a consequence of Theorem 3 :

Corollary 5 The roots of $H_{n}(x)$ are real and interlace those of $H_{n+1}(x)$ for $n>0$.

Proof The polynomials $H_{n}(x)$ are clearly not orthogonal, so the proof of the interlacing phenomenon does not follow from classical results, nor directly from the results of [10]. Nevertheless an elementary induction argument works, which we also omit.

It is also curious that the recursion (126) seems to define a sequence of rational functions with nontrivial denominators. We are unaware of a general theory of such recurrences which actually define Sturm sequences of polynomials.

\section{Transformation rules}

Before we proceed with the evaluation of the determinant $H_{n}^{(2,1)}(x)$, we would like to collect the information we already have about various trace calculations to automate this process to some extent. In the course of the calculations involved in the $(3,1)$-case, we have had to evaluate traces such as

$$
\operatorname{Tr}\left(A_{n}^{-1}\left[p(x) a_{i+j+1}\right]_{0 \leq i, j \leq n}\right), \quad \operatorname{Tr}\left(A_{n}^{-1}\left[q(x)(i+j) a_{i+j+1}\right]_{0 \leq i, j \leq n}\right), \quad \operatorname{Tr}\left(A_{n}^{-1}\left[r(x) c_{i+j}\right]_{0 \leq i, j \leq n}\right)
$$


for polynomial coefficients $p(x), q(x), r(x)$. These traces are reduced to calculating traces of the form

$$
\operatorname{Tr}\left(A_{n}^{-1}\left[a_{i+j+1}\right]\right), \quad \operatorname{Tr}\left(A_{n}^{-1}\left[(i+j) a_{i+j+1}\right]\right), \quad \operatorname{Tr}\left(A_{n}^{-1}\left[c_{i+j}\right]\right)
$$

etc., and then use linearity of the trace. Furthermore, the calculations do not use the explicit form of the polynomials $a_{k}$. We can go one step further: when we apply the operator $\operatorname{Tr}\left(A_{n}^{-1} *\right)$ to various terms of an identity linearly, we can then multiply through the resulting transformed identity by $H_{n}$ to obtain a relationship between various determinants related to $H_{n}$, as we did in a number of cases in the computation of the (3,1)-case. In Table 1 we summarize the effect of this transformation on various terms that may appear in an identity.

\begin{tabular}{|l|l|l|}
\hline$d_{x} a_{n} \rightarrow d_{x} H_{n}$ & & \\
\hline \hline & $n a_{n-1} \rightarrow 0$ & $c_{n-1} \rightarrow 0$ \\
\hline$a_{n} \rightarrow(n+1) H_{n}$ & $n a_{n} \rightarrow n(n+1) H_{n}$ & $c_{n} \rightarrow(2 n+1) a_{0} H_{n}$ \\
\hline$a_{n+1} \rightarrow K_{n}$ & $n a_{n+1} \rightarrow 2 n K_{n}$ & $c_{n+1} \rightarrow 2 a_{0} K_{n}+2 n a_{1} H_{n}$ \\
\hline $\begin{array}{l}a_{n+2} \rightarrow M_{n}+N_{n} \\
(n>0)\end{array}$ & $\begin{array}{l}n a_{n+2} \rightarrow 2(n-1) M_{n}+2 n N_{n} \\
(n>0)\end{array}$ & \\
\hline
\end{tabular}

Table 1: Some linear transformation rules.

In Table $1, a_{n}=a_{n}(x), n=0,1, \ldots$ is a sequence of polynomials, $c_{n}$ are the convolution polynomials defined by

$$
c_{n}=\sum_{i=0}^{n} a_{i} a_{n-i}
$$

and $H_{n}, K_{n}, M_{n}, N_{n}$ are the following $(n+1) \times(n+1)$ determinants defined by the $a_{k}$ 's:

$$
\begin{gathered}
H_{n}=\operatorname{det}\left[\begin{array}{ccccc}
a_{0} & a_{1} & a_{2} & \ldots & a_{n} \\
a_{1} & a_{2} & a_{3} & \ldots & a_{n+1} \\
\vdots & & & & \vdots \\
a_{n} & a_{n+1} & \ldots & & a_{2 n}
\end{array}\right] \quad K_{n}=\operatorname{det}\left[\begin{array}{cccccc}
a_{0} & a_{1} & \ldots & a_{n-1} & a_{n+1} \\
a_{1} & a_{2} & \ldots & a_{n} & a_{n+2} \\
\vdots & & \ddots & & \\
a_{n-1} & a_{n} & \ldots & a_{2 n-2} & a_{2 n} \\
a_{n} & a_{n+1} & \ldots & a_{2 n-1} & a_{2 n+1}
\end{array}\right] \\
M_{n}=\operatorname{det}\left[\begin{array}{cccccc}
a_{0} & a_{1} & \ldots & a_{n-2} & a_{n+1} & a_{n} \\
a_{1} & a_{2} & \ldots & a_{n-1} & a_{n+2} & a_{n+1} \\
\vdots & & \ddots & & & \\
a_{n-1} & a_{n} & \ldots & a_{2 n-3} & a_{2 n} & a_{2 n-1} \\
a_{n} & a_{n+1} & \ldots & a_{2 n-2} & a_{2 n+1} & a_{2 n}
\end{array}\right]
\end{gathered}
$$




$$
N_{n}=\operatorname{det}\left[\begin{array}{cccccc}
a_{0} & a_{1} & \ldots & a_{n-2} & a_{n-1} & a_{n+2} \\
a_{1} & a_{2} & \ldots & a_{n-1} & a_{n} & a_{n+3} \\
\vdots & & \ddots & & & \\
a_{n-1} & a_{n} & \ldots & a_{2 n-3} & a_{2 n-2} & a_{2 n+1} \\
a_{n} & a_{n+1} & \ldots & a_{2 n-2} & a_{2 n-1} & a_{2 n+2}
\end{array}\right]
$$

From these rules we obtain a few known applications that are not connected with the determinants we are evaluating. We give a few simple examples.

\section{Example}

Recall that a polynomial sequence $\left\{a_{k}\right\}_{k \geq 0}$ is said to be an Appell set if $d_{x} a_{n}=n a_{n-1}$. If $B_{n}, \mathcal{H}_{n}, T_{n}, P_{n}$ are the Bernoulli, Hermite, Chebyshev, and Legendre polynomials, respectively, then for any complex $a \neq 0$,

$$
\left\{B_{n}(x)\right\}, \quad\left\{(2 a)^{-n} \mathcal{H}_{n}(a x)\right\}, \quad\left\{\left(x^{2}-a^{2}\right)^{\frac{n}{2}} T_{n}\left(\frac{x}{\sqrt{x^{2}-a^{2}}}\right)\right\}, \quad\left\{\left(x^{2}-a^{2}\right)^{\frac{n}{2}} P_{n}\left(\frac{x}{\sqrt{x^{2}-a^{2}}}\right)\right\},
$$

are all well-known examples of Appell sets [3, 14]. Starting with the defining identity

$$
d_{x} a_{n}=n a_{n-1}
$$

and applying the transformations $d_{x} a_{n} \rightarrow d_{x} H_{n}$ and $n a_{n-1} \rightarrow 0$ from Table 1 , we see that $d_{x} H_{n}=0$, and therefore the Hankel determinants of Appell polynomials are independent of $x$.

\section{Example}

For a sequence of polynomials $a_{n}$ satisfying

$$
x d_{x} a_{n}=n a_{n}+c n a_{n-1}
$$

for some constant $c$, we make the replacements $d_{x} a_{n} \rightarrow d_{x} H_{n}, n a_{n} \rightarrow n(n+1) H_{n}$ and $n a_{n-1} \rightarrow 0$ from Table 1 to find that the corresponding Hankel determinant $H_{n}$ satisfies

$$
x d_{x} H_{n}=n(n+1) H_{n} .
$$

This is integrable with

$$
H_{n}(x)=c_{n} \cdot x^{n(n+1)} .
$$

For $c=-1$ in (128) we get the Laguerre polynomials $L_{n}$ with $L_{0}=1, L_{1}=1-x$. After simple row operations, $c_{n}=H_{n}(1)$ has a known product evaluation as

$$
c_{n}=\operatorname{det}\left[\frac{1}{(i+j) !}\right]_{0 \leq i, j \leq n}=2^{-n^{2}}(-1)^{\frac{n(n+1)}{2}} \prod_{i=1}^{n} \frac{1}{1^{2} \cdot 3^{2} \cdots(2 i-1)^{2}} .
$$


For $c=1$ in (128) we get the so-called derangement polynomials

$$
D_{n}(x)=\sum_{i=0}^{n}(-1)^{i} \frac{n !}{i !} x^{n-i}
$$

for which the constant of integration is

$$
c_{n}=\prod_{i=1}^{n} i !^{2}
$$

as found in $[12]$.

\section{Example}

Legendre polynomials satisfy

$$
\left(x^{2}-1\right) d_{x} P_{n}=n x P_{n}-n P_{n-1}
$$

with $P_{0}=1, P_{1}=x$. Therefore

$$
\left(x^{2}-1\right) d_{x} H_{n}=x n(n+1) H_{n}
$$

and

$$
H_{n}=c_{n} \cdot\left(x^{2}-1\right)^{\frac{n(n+1)}{2}} .
$$

$H_{n}$ can be evaluated at $x=0$, giving

$$
c_{n}=2^{-n^{2}}
$$

\section{Example}

In general, for any family of polynomials $\left\{a_{k}\right\}_{k \geq 0}$ such that

$$
d_{x} a_{n}, \quad a_{n}, \quad n a_{n}, \quad n a_{n-1}, \quad c_{n}, \quad c_{n-1}
$$

satisfy a linear identity with fixed polynomial coefficients (where $c_{n}$ is the convolution defined in (127)), the corresponding Hankel determinant is the solution to a simple separable first-order linear equation.

Now we return to the determinants $H_{n}^{(\beta, \alpha)}$ and consider the $(2,1)$-case. 


\section{The $(2,1)$-case}

Now we let

$$
\begin{aligned}
a_{k} & =a_{k}^{(2,1)}(x)=\sum_{m=0}^{k}\left(\begin{array}{c}
2 k+1-m \\
k-m
\end{array}\right) x^{m} \\
c_{k} & =\sum_{i=0}^{k} a_{i} a_{k-i} \\
H_{n} & =H_{n}^{(2,1)}(x)=\operatorname{det}\left[a_{i+j}(x)\right]_{0 \leq i, j \leq n} .
\end{aligned}
$$

Theorem 4 Let the polynomials $a_{k}=a_{k}^{(2,1)}(x)$ and $H_{n}=H_{n}^{(2,1)}(x)$ are as defined in (129). Then $H_{n}$ satisfies the differential equation

$$
x(x-2) d_{x}^{2} y+(2 x-1) d_{x} y-n(n+1) y=0 .
$$

The proof of Theorem 4 mimics the steps of the $(3,1)$-case. The analogues of the first two identities in Lemma 1 and Lemma 2 of the $(3,1)$-case are now as follows:

Lemma 6 Suppose $a_{k}$ and $c_{k}$ are as defined in (129). Then

$$
2 x(x-2) d_{x} a_{n}-(n+1) a_{n+1}+(4 n+2(x+1)) a_{n}-(x-1) c_{n}+4(x-1) c_{n-1}=0 .
$$

Lemma 7 Suppose $a_{k}$ and $c_{k}$ are as defined in (129). Then

$$
\begin{aligned}
(n x+2(x+1)) a_{n+2}-\left(2 x(x+2) n+2\left(2 x^{2}+3 x+4\right)\right) a_{n+1} \\
+4 x^{2}(2 n+3) a_{n}+(x-1)(x-2) c_{n+1}-4(x-1)(x-2) c_{n}=0 .
\end{aligned}
$$

The proofs are given in Appendix II.

Applying the transformations in Table 1 to the identities in these two Lemmas, we immediately obtain the pair of identities

$$
\begin{aligned}
& 2 x(x-2) d_{x} H_{n}-(2 n+1) K_{n} \\
& +4 n(n+1) H_{n}+2(x+1)(n+1) H_{n}-(x-1)(2 n+1) H_{n}=0, \\
& x\left(2(n-1) M_{n}+2 n N_{n}\right)+2(x+1)\left(M_{n}+N_{n}\right)-2 x(x+2)\left(2 n K_{n}\right) \\
& \quad+2\left(2 x^{2}+3 x+4\right) K_{n}+8 x^{2} n(n+1) H_{n}+12 x^{2}(n+1) H_{n} \\
& \quad+(x-1)(x-2)\left(2 K_{n}+2 n(x+3) H_{n}\right)-4(x-1)(x-2)(2 n+1) H_{n}=0 .
\end{aligned}
$$

These can be rewritten as

$$
\begin{aligned}
& 2 x(x-2) d_{x} H_{n}+\left(x+3+8 n+4 n^{2}\right) H_{n}-(2 n+1) K_{n}=0, \\
& \left(2 n x^{3}+\left(8 n^{2}+12 n+8\right) x^{2}+(12 n+10) x-4 n-8\right) H_{n} \\
& -\left((2+4 n) x^{2}+(12+8 n) x+4\right) K_{n}+2(n x+1) M_{n}+2((n+1) x+1) N_{n}=0 .
\end{aligned}
$$


First we use identity (25) to rewrite $K_{n}$ as a trace in (133). Differentiating, we follow through calculations of traces similar to the derivation of $d_{x}^{2} H_{n}$ of the $(3,1)$-case to obtain

$$
\begin{gathered}
2(x-2)^{2} x^{2}(1+n x) d_{x}^{2} H_{n} \\
+2(x-2) x\left(3+10 n+4 n^{2}+3 x+9 n x+12 n^{2} x+4 n^{3} x+4 n x^{2}+2 n^{2} x^{2}\right) d_{x} H_{n} \\
+\left(10+49 n+78 n^{2}+44 n^{3}+8 n^{4}+\left(4+34 n+86 n^{2}+98 n^{3}+48 n^{4}+8 n^{5}\right) x\right. \\
\left.+\left(1+8 n+14 n^{2}+8 n^{3}\right) x^{2}+\left(2 n-2 n^{3}\right) x^{3}\right) H_{n} \\
-(2 n+1)\left(1+2 n+2 n x+2 n^{2} x\right) N_{n}=0 .
\end{gathered}
$$

Combining (134) and the expression (133) for $d_{x} H_{n}$, we obtain

$$
\begin{gathered}
2(x-2) x(n x+1)\left(x(x-2) d_{x}^{2} y+(2 x-1) d_{x} y-n(n+1) y\right)= \\
(2 n+1)\left(1+2 n+2 n x+2 n^{2} x\right)\left(N_{n}-(4+2 n+x) K_{n}\right. \\
\left.+\left(2+5 n+2 n^{2}+3 x+2 n x\right) H_{n}\right) .
\end{gathered}
$$

\subsection{Product form evaluations at special $x$ for the $(2,1)$-case}

At this point, we can evaluate $H_{n}(x)$ at special points $x$ easily. At $x=0$, we get from (133) and (134)

$$
\begin{aligned}
K_{n} & =\frac{3+8 n+4 n^{2}}{2 n+1} H_{n} \\
N_{n} & =\frac{10+49 n+78 n^{2}+44 n^{3}+8 n^{4}}{(2 n+1)^{2}} H_{n} \\
M_{n} & =-\left(3 n+2 n^{2}\right) H_{n} .
\end{aligned}
$$

Using (75) and that $H_{0}=H_{1}=1$ for $x=0$, we obtain the recursion for $\frac{H_{n}}{H_{n-1}}$ as

$$
\frac{H_{n+1}}{H_{n}}=\frac{H_{n}}{H_{n-1}}
$$

and therefore

$$
H_{n}(0)=1
$$

as is well known. At $x=2$

$$
\begin{aligned}
K_{n} & =\frac{5+8 n+4 n^{2}}{2 n+1} H_{n} \\
N_{n} & =\frac{22+33 n+20 n^{2}+4 n^{3}}{2 n+1} H_{n} \\
M_{n} & =-\frac{n\left(7+8 n+4 n^{2}\right)}{(2 n+1)} H_{n}
\end{aligned}
$$


and $H_{0}=1, H_{1}=-3$. From $(75)$

$$
H_{n+1} H_{n-1}=\frac{(2 n-1)(2 n+3)}{(2 n+1)^{2}} H_{n}^{2}
$$

and therefore

$$
H_{n}(2)=(-1)^{n}(2 n+1) .
$$

After the evaluations at special points, we now return to the proof of the differential equation for the $2 n+1$ case. By (135), it suffices to prove the identity

$$
N_{n}-(4+2 n+x) K_{n}+\left(2+5 n+2 n^{2}+3 x+2 n x\right) H_{n}=0 .
$$

This is the determinantal form of the third identity for the $(2,1)$-case. The left hand side of (141) is a determinant as in (99) and (100) of the $(3,1)$-case. The problem is again to show the existence of weights $w_{n, 0}, w_{n, 1}, \ldots, w_{n, n+2}$ satisfying the third identity (101) and (102) where in this case the requirements are

$$
\begin{aligned}
w_{n, n+2} & =p_{n}(x)=1 \\
w_{n, n+1} & =q_{n}(x)=-(4+2 n+x) \\
w_{n, n} & =r_{n}(x)=2+5 n+2 n^{2}+3 x+2 n x .
\end{aligned}
$$

By experimentation, we found that these weights are explicitly given by

$$
\begin{aligned}
w_{n, j}=(-1)^{n+j}\left\{2\left(\begin{array}{c}
n+j+1 \\
n-j+1
\end{array}\right)+\left(\begin{array}{c}
n+j+1 \\
n-j+2
\end{array}\right)\right. & \left.+\left(2\left(\begin{array}{c}
n+j+1 \\
n-j
\end{array}\right)+\left(\begin{array}{l}
n+j+1 \\
n-j+1
\end{array}\right)\right) x\right\}
\end{aligned}
$$

for $i=0,1, \ldots, n-1$, and $w_{n, n}, w_{n, n+1}, w_{n, n+2}$ as in (142). The resulting identities are simpler than the $(3,1)$-case, and in fact a combination of paper-and-pencil and automatic identity provers can be used to prove that these are correct. However we can do better, and we use this case as another exercise for the application of the method of the existence of the weights that we used in the $(3,1)$-case.

We need a lemma similar to Lemma 5.

Lemma 8 Suppose $a_{k}(x)$ is as defined in (9) and $f$ is as in (104). Then

$$
f(x, y)=\frac{t}{(x-2) y t+1-2 x y}
$$

where

$$
t=\sum_{k \geq 0} \frac{(2 k) !}{(k+1) ! k !} y^{k}=1+y+2 y^{2}+5 y^{3}+\cdots
$$

satisfies $t^{2} y=t-1$. 
The proof of Lemma 8 can be found in Appendix I.

Let $t$ be the power series in $y$ given in Lemma 8. Then there is a nontrivial polynomial $Q_{0}=Q_{0}(y)$ of degree $n+1$ such that

$$
t Q_{0}=Q_{1}+y^{2 n+3} \Psi_{0}
$$

where $Q_{1}=Q_{1}(y)$ is a polynomial of degree $n+1$, and $\Psi_{0}=\Psi_{0}(y)$ is a power series in $y$; i.e. the coefficients of $y^{k}$ in $t Q_{0}$ vanish for $n+2 \leq k \leq 2 n+2$. Such a nontrivial $Q_{0}$ exists because there are $n+2$ coefficients and only $n+1$ linear homogeneous equations these coefficients need to satisfy. In the next step, put

$$
Q_{2}=(x-2) y Q_{1}+(1-2 x y) Q_{0}
$$

Then $Q_{2}=Q_{2}(x, y)$ is a polynomial in $x$ and $y$ of $y$-degree $n+2$. All three polynomials $Q_{0}, Q_{1}, Q_{2}$ are nontrivial. We claim that the coefficients of $Q_{2}$ are the weights we want. In other words, the coefficients of the terms $y^{n+2}$ through $y^{2 n+2}$ in $f Q_{2}$ vanish. Writing (144) in the form

$$
f(x, y)((x-2) y t+1-2 x y)=t
$$

and multiplying through by $Q_{0}$, we get

$$
f(x, y)\left((x-2) y\left(Q_{1}+y^{2 n+3} \Psi_{0}\right)+(1-2 x y) Q_{0}\right)=Q_{1}+y^{2 n+3} \Psi_{0}
$$

or

$$
f(x, y) Q_{2}=Q_{1}+y^{2 n+3} \Psi_{0}-f(x, y)(x-2) y y^{2 n+3} \Psi_{0}
$$

and therefore

$$
f(x, y) Q_{2}=Q_{1}+y^{2 n+3}\left(\Psi_{0}-f(x, y)(x-2) y \Psi_{0}\right)
$$

which means that

$$
f Q_{2}=Q_{1}+y^{2 n+3} \Psi_{1}
$$

where $\Psi_{1}=\Psi(y)$ is a power series in $y$. This last statement (147) is equivalent to

$$
\sum_{j=0}^{n+2} \mathcal{C}_{n+2-j}\left(Q_{2}\right) a_{i+j}=0
$$

for $i=0,1, \ldots, n$, where by $\mathcal{C}_{k}(\Psi)$ we denote the coefficient of the term $y^{k}$ in a power series $\Psi$. Thus (102) holds with

$$
w_{n, j}=\mathcal{C}_{n+2-j}\left(Q_{2}\right)
$$

for $j=0,1, \ldots, n+2$.

Therefore

$$
\mathcal{C}_{0}\left(Q_{2}\right) N_{n}+\mathcal{C}_{1}\left(Q_{2}\right) K_{n}+\mathcal{C}_{2}\left(Q_{2}\right) H_{n}=0
$$


This identity is not trivial, for otherwise we would have a nontrivial linear relationship among the $n+1$ columns $v_{0}, v_{1}, \ldots, v_{n}$ of $H_{n}$. But we have already shown by the evaluations in (138), (140) that $H_{n}$ does not identically vanish.

Now we need verify the three special values in (142), i.e. for some nonzero constant $\alpha$,

$$
\begin{aligned}
& \mathcal{C}_{0}\left(Q_{2}\right)=\alpha p_{n} \\
& \mathcal{C}_{1}\left(Q_{2}\right)=\alpha q_{n} \\
& \mathcal{C}_{2}\left(Q_{2}\right)=\alpha r_{n}
\end{aligned}
$$

where $p_{n}, q_{n}, r_{n}$ are as defined in (142).

Rewrite (149) in terms of $\mathcal{C}_{0}\left(Q_{0}\right), \mathcal{C}_{1}\left(Q_{0}\right), \mathcal{C}_{2}\left(Q_{0}\right)$ which are pure constants, independent on $x$ and $y$. Comparing coefficients in (145) and (146), we obtain

$$
\begin{aligned}
& \mathcal{C}_{0}\left(Q_{1}\right)=\mathcal{C}_{0}\left(Q_{0}\right) \\
& \mathcal{C}_{1}\left(Q_{1}\right)=\mathcal{C}_{1}\left(Q_{0}\right)+\mathcal{C}_{0}\left(Q_{0}\right) \\
& \mathcal{C}_{0}\left(Q_{2}\right)=\mathcal{C}_{0}\left(Q_{0}\right) \\
& \mathcal{C}_{1}\left(Q_{2}\right)=(x-2) \mathcal{C}_{0}\left(Q_{1}\right)-2 x \mathcal{C}_{0}\left(Q_{0}\right)+\mathcal{C}_{1}\left(Q_{0}\right) \\
& \mathcal{C}_{2}\left(Q_{2}\right)=(x-2) \mathcal{C}_{1}\left(Q_{1}\right)-2 x \mathcal{C}_{1}\left(Q_{0}\right)+\mathcal{C}_{2}\left(Q_{0}\right) .
\end{aligned}
$$

Therefore

$$
\begin{aligned}
& \mathcal{C}_{0}\left(Q_{2}\right)=\mathcal{C}_{0}\left(Q_{0}\right) \\
& \mathcal{C}_{1}\left(Q_{2}\right)=\mathcal{C}_{1}\left(Q_{0}\right)-(x+2) \mathcal{C}_{0}\left(Q_{0}\right) \\
& \mathcal{C}_{2}\left(Q_{2}\right)=\mathcal{C}_{2}\left(Q_{0}\right)-(x+2) \mathcal{C}_{1}\left(Q_{0}\right)+(x-2) \mathcal{C}_{0}\left(Q_{0}\right)
\end{aligned}
$$

and (149) becomes

$$
\begin{aligned}
\mathcal{C}_{0}\left(Q_{0}\right) & N_{n}+ \\
& \left(\mathcal{C}_{1}\left(Q_{0}\right)-(x+2) \mathcal{C}_{0}\left(Q_{0}\right)\right) K_{n}+ \\
& \left(\mathcal{C}_{2}\left(Q_{0}\right)-(x+2) \mathcal{C}_{1}\left(Q_{0}\right)+(x-2) \mathcal{C}_{0}\left(Q_{0}\right)\right) H_{n}=0 .
\end{aligned}
$$

Using the expressions for $K_{n}$ and $N_{n}$ in terms of $H_{n}$ at $x=0$ and $x=2$ from (136) and (139)

$$
\begin{aligned}
\left(10+49 n+78 n^{2}\right. & \left.+44 n^{3}+8 n^{4}\right) \mathcal{C}_{0}\left(Q_{0}\right) \\
+(3+8 n & \left.+4 n^{2}\right)(2 n+1)\left(\mathcal{C}_{1}\left(Q_{0}\right)-2 \mathcal{C}_{0}\left(Q_{0}\right)\right) \\
+ & (2 n+1)^{2}\left(\mathcal{C}_{2}\left(Q_{0}\right)-2 \mathcal{C}_{1}\left(Q_{0}\right)-2 \mathcal{C}_{0}\left(Q_{0}\right)\right)=0 \\
\left(22+33 n+20 n^{2}\right. & \left.+4 n^{3}\right) \mathcal{C}_{0}\left(Q_{0}\right) \\
+(5+8 n & \left.+4 n^{2}\right)\left(\mathcal{C}_{1}\left(Q_{0}\right)-4 \mathcal{C}_{0}\left(Q_{0}\right)\right) \\
+ & (2 n+1)\left(\mathcal{C}_{2}\left(Q_{0}\right)-4 \mathcal{C}_{1}\left(Q_{0}\right)\right)=0 .
\end{aligned}
$$


The parametric solutions are

$$
\begin{aligned}
& \mathcal{C}_{2}\left(Q_{0}\right)=n(2 n+1) \alpha \\
& \mathcal{C}_{1}\left(Q_{0}\right)=-2(n+1) \alpha \\
& \mathcal{C}_{0}\left(Q_{0}\right)=\alpha .
\end{aligned}
$$

Going back to the system (152)

$$
\begin{aligned}
& \mathcal{C}_{0}\left(Q_{2}\right)=\alpha \\
& \mathcal{C}_{1}\left(Q_{2}\right)=-(4+2 n+x) \alpha \\
& \mathcal{C}_{2}\left(Q_{2}\right)=\left(2+5 n+2 n^{2}+3 x+2 n x\right) \alpha .
\end{aligned}
$$

With $\alpha=1$, these are exactly the weights $w_{n, n+2}=p_{n}, w_{n, n+1}=q_{n}, w_{n, n}=r_{n}$ as claimed in (142).

\subsection{The differential equation solution for the $(2,1)$-case}

We have the differential equation (130) satisfied by the Hankel determinants $H_{n}$ for the $(2,1)$-case. We obtain a power series solution to (130) as

$$
y(x)=C_{n} \sum_{i=0}^{n} \frac{(n+i) ! 2^{i}(x-2)^{i}}{(n-i) !(2 i+1) !}
$$

where he constant of integration $C_{n}=H_{n}(2)$ is given by (140). Therefore

Theorem 5 Suppose $a_{k}(x)$ is defined by (9) and $H_{n}(x)=H_{n}^{(2,1)}(x)=\operatorname{det}\left[a_{i+j}(x)\right]_{0 \leq i, j \leq n}$. Then

$$
H_{n}(x)=(-1)^{n}(2 n+1) \sum_{i=0}^{n} \frac{(n+i) ! 2^{i}(x-2)^{i}}{(n-i) !(2 i+1) !} .
$$

Evaluating (156) at $x=0$ and at $x=1$ we obtain

\section{Corollary 6}

$$
\begin{aligned}
& \operatorname{det}\left[\left(\begin{array}{c}
2(i+j)+1 \\
i+j
\end{array}\right)\right]_{0 \leq i, j \leq n}=(-1)^{n}(2 n+1) \sum_{i=0}^{n} \frac{(n+i) !(-4)^{i}}{(n-i) !(2 i+1) !} \\
& \operatorname{det}\left[\left(\begin{array}{c}
2(i+j)+2 \\
i+j
\end{array}\right)\right]_{0 \leq i, j \leq n}=(-1)^{n}(2 n+1) \sum_{i=0}^{n} \frac{(n+i) !(-2)^{i}}{(n-i) !(2 i+1) !}
\end{aligned}
$$

We remark that the known product forms for the Hankel determinant evaluations in this corollary follow by writing the series in (157) as a hypergeometric series

$$
{ }_{2} F_{1}\left[\begin{array}{cc}
-n, n+1 & ; 1 \\
1 &
\end{array}\right]
$$


and applying the Chu-Vandermonde summation formula, respectively by writing the series in (158) as a geometric series

$$
{ }_{2} F_{1}\left[\begin{array}{cc}
-n, n+1 & ; \frac{1}{2}
\end{array}\right]
$$

and applying Bailey's summation

$$
{ }_{2} F_{1}\left[\begin{array}{c}
a, 1-a \\
b
\end{array} ; \frac{1}{2}\right]=\frac{\Gamma\left(\frac{b}{2}\right) \Gamma\left(\frac{1}{2}+\frac{b}{2}\right)}{\Gamma\left(\frac{a}{2}+\frac{b}{2}\right) \Gamma\left(\frac{1}{2}-\frac{a}{2}+\frac{b}{2}\right)} .
$$

An interesting point about Corollary 6 is that the right hand side of (157) is a complicated way of writing 1 , while the right hand side of (158) evaluates to the simple expression

$$
(-1)^{\frac{n(n+1)}{2}}
$$

Alternate derivations of the evaluation (159) for the determinant in (158) were communicated to us by Ira Gessel and Christian Krattenthaler. A generalization of the determinant in (158) can be found in Corollary 8 below.

It is also interesting that the determinants $H_{n}^{(2,1)}(x)$ are orthogonal polynomials:

Corollary 7 The $(n+1) \times(n+1)$ Hankel determinants $H_{n}(x)=H_{n}^{(2,1)}(x)$ form an orthogonal family of polynomials.

Proof We have

$$
H_{0}(x)=1, \quad H_{1}(x)=1-2 x
$$

and for $n \geq 2$,

$$
H_{n}(x)=2(1-x) H_{n-1}(x)-H_{n-2}(x)
$$

which can be verified directly from the explicit formula in (156) or using the differential equation (130). Orthogonality now follows from Favard's theorem.

In this case the generating function of the $H_{n}(x)$ is

$$
\frac{1-y}{1-2(1-x) y+y^{2}}=\sum_{n \geq 0} H_{n}(x) y^{n} .
$$

Comparing this last expression with the generating function

$$
\frac{1}{1-2 x y+y^{2}}=\sum_{n \geq 0} U_{n}(x) y^{n}
$$

of the Chebyshev polynomials of the second kind $U_{n}(x)$, we have the expansion

$$
H_{n}(x)=U_{n}(1-x)-U_{n-1}(1-x)
$$

THE ELECTRONIC JOURNAL OF COMBINATORICS 15 (2008), \#R6 
In fact we can prove more. From the identity

$$
\sum_{m=0}^{k}\left(\begin{array}{c}
2 k+2 \\
k-m
\end{array}\right) x^{m}=\sum_{m=0}^{k}\left(\begin{array}{c}
2 k+1-m \\
k-m
\end{array}\right)(x+1)^{m}=a_{k}^{(2,1)}(x+1)
$$

and the differential equation (130) for $H_{n}^{(2,1)}(x)$, we obtain both the evaluation of the Hankel determinant of the polynomials

$$
a_{k}(x)=\sum_{m=0}^{k}\left(\begin{array}{c}
2 k+2 \\
k-m
\end{array}\right) x^{m}
$$

and an alternate expression for $H_{n}^{(2,1)}(x)$ itself.

Corollary 8 Suppose the polynomials $a_{k}(x)$ are as defined in (163) and

$$
H_{n}(x)=\operatorname{det}\left[a_{i+j}(x)\right]_{0 \leq i, j \leq n}
$$

Then $H_{n}(x)$ satisfies Jacobi's differential equation

$$
\left(x^{2}-1\right) d_{x}^{2} y+(2 x+1) d_{x} y-n(n+1) y=0
$$

and therefore in terms of the Jacobi polynomials

$$
\begin{aligned}
H_{n}(x) & =(-4)^{n} \frac{(n !)^{2}}{(2 n) !} P_{n}^{\left(\frac{1}{2},-\frac{1}{2}\right)}(x), \\
H_{n}^{(2,1)}(x) & =(-4)^{n} \frac{(n !)^{2}}{(2 n) !} P_{n}^{\left(\frac{1}{2},-\frac{1}{2}\right)}(x-1) .
\end{aligned}
$$

\section{Remarks}

When we started to look at Hankel determinants of $A_{n}=\left[a_{i+j}\right]_{0 \leq i, j \leq n}$ with entries $a_{k}=$ $\left(\begin{array}{c}3 k+1 \\ k\end{array}\right)$, it looked like an almost product could account for the combination of small and large prime factors that were showing up in the data. At the time, we also thought that whatever was moving the $(3,1)$-case off the pure product formulas of the $(3,0)$ and $(3,2)$ cases would also explain the $(3,3)$-case, and the $(3,4)$-case, and so on. It might also get at the $(4,1)$-case, and all the rest of the $(\beta, \alpha)$-cases.

This section is a brief discussion of what did and did not work out. We started with the observation that if you define $a_{k}(x)$ by $(7)$ then

$$
a_{k}(1)=\left(\begin{array}{c}
3 k+2 \\
k
\end{array}\right)
$$

so that

$$
H_{n}^{(3,2)}=H_{n}^{(3,1)}(1)
$$


has a product form evaluation. What we wanted was the value of $H_{n}^{(3,1)}(x)$ not at $x=1$ but at $x=0$. Experimenting with the expansion of $H_{n}^{(3,1)}(x)$ at various points, we found that at $x=1$ and at $x=3$ the Taylor coefficients of $H_{n}^{(3,1)}(x)$ had small prime factors. We called $x=1$ and $x=3$ round points.

We also found several Hankel determinants that had similar behavior but we had trouble finding a consistent explanation for this phenomenon. In others, such as $H_{n}^{(3,0)}$, the coefficients were somewhat round but not round enough to allow us to find a simple power series expansion.

When we tried to determine the common elements of these different determinants, differential equations came to mind because hypergeometric series such as (8) clearly have a differential equation. In retrospect, the explanation of why $x=1$ and $x=3$ are good places to expand $H_{n}^{(3,1)}$ is partially explained by examining the form of its differential equation. For instance (14) can be written in the form

$$
(x-3)^{2} d_{x}^{2} y+2(n+2)(x-3) d_{x} y-3 n(n+1) y=-2(x-3) d_{x}^{2} y-3 d_{x} y .
$$

This form is significant because the operator on the left hand side of $(165)$ takes $(x-3)^{k}$ to $(x-3)^{k}$ times a polynomial in $n$ and $k$ and the operator on the right takes $(x-3)^{k}$ to $(x-3)^{k-1}$ times a polynomial in $n$ and $k$. This means that the differential equation defines a two-term recursion on successive coefficients of the expansion of $H_{n}^{(3,1)}(x)$ around $(x-3)$. So the power series is easily shown to be hypergeometric.

Differential equations can also explain the "near" round behavior seen in some cases, such as the $H_{n}^{(3,0)}$. However, the existence of a differential equation would not provide any input on why the constant of integration such as $H_{n}^{(3,0)}(3)$ was round. Consequently it was a nice bonus when, during the proof of the differential equation, we realized that we could prove product formulas for these kinds of determinants (e.g., (83) in Corollary 1, (90) in Corollary 2, (95) in Corollary 3; (168), (169), and (172) in Section 8).

Once differential equations entered the picture, it was easy to guess the differential equations for many of these Hankel determinants and obtain strong experimental evidence for their correctness, but we did not find a general pattern, even for the families $H_{n}^{(\beta, \alpha)}$. It is doubtful that such a pattern exists. For example, the (unproven) differential equation for the (3,2)-case is fourth order and quite mysteriously complex (see Figure 1). This differential equation has been tested for $H_{n}^{(3,2)}(x)$ where $n=1,2, \ldots, 75$. We did not find a differential equation for $(3,3)$ or for any $(3, \alpha)$ with $\alpha \geq 3$.

Now the actual proof of the differential equation for a determinant like $H_{n}^{(3,1)}(x)$ is an elusive creature. The straightforward evaluation of the derivative yields a sum of $n+1$ determinants, each one of which is badly behaved at $x=3$. That was a disturbing development, and we still had to differentiate each of these determinants themselves to get at the second order differential equation.

In order to approach the problem systematically, we wrote the derivative of a Hankel 


$$
\begin{aligned}
& 2(3 x-1)^{2}(x-3)^{3} x\left(4(n+2)(2 n+1) x^{2}+\left(8 n^{2}+20 n+11\right) x-1\right) d_{x}^{4} y \\
& +(x-3)^{2}(3 x-1)\left(12(n+2)(2 n+1)(8 n+27) x^{4}\right. \\
& -3\left(128 n^{3}+568 n^{2}+724 n+161\right) x^{3} \\
& \left.-\left(576 n^{3}+3016 n^{2}+4756 n+2269\right) x^{2}+\left(72 n^{2}+252 n+319\right) x-15\right) d_{x}^{3} y \\
& +3(x-3)\left(12\left(8 n^{4}+118 n^{3}+427 n^{2}+533 n+174\right) x^{5}\right. \\
& -\left(736 n^{4}+6704+19628 n^{2}+21289 n+5814\right) x^{4} \\
& +\left(800 n^{4}+2944 n^{3}+564 n^{2}-7580 n-7078\right) x^{3} \\
& +2\left(816 n^{4}+6744 n^{3}+19358 n^{2}+23069 n+9809\right) x^{2} \\
& \left.-6\left(108 n^{3}+540 n^{2}+972 n+679\right) x+15(9 n+20)\right) d_{x}^{2} y \\
& -3\left(12\left(16 n^{5}+62 n^{4}-7 n^{3}-293 n^{2}-378 n-120\right) x^{5}\right. \\
& -\left(960 n^{5}+3104 n^{4}-3080 n^{3}-20993 n^{2}-23419 n-6450\right) x^{4} \\
& +4\left(144 n^{5}-4 n^{4}-2300 n^{3}-4657 n^{2}-2040 n+838\right) x^{3} \\
& +\left(1728 n^{5}+6624 n^{4}-5832 n^{3}-556990 n^{2}-80626 n-36004\right) x^{2} \\
& \left.+12\left(318 n^{4}+1995 n^{3}+4670 n^{2}+4928 n+2058\right) x-\left(783 n^{2}+3105 n+3102\right)\right) d_{x} y \\
& -3 n(n+1)\left(12\left(12 n^{4}+68 n^{3}+137 n^{2}+113 n+30\right) x^{4}\right. \\
& -\left(864 n^{4}+4488 n^{3}+8158 n^{2}+5887 n+1222\right) x^{3} \\
& +\left(720 n^{4}+2304 n^{3}+280 n^{2}-4547 n-3388\right) x^{2} \\
& \left.+3\left(576 n^{4}+3816 n^{3}+9182 n^{2}+9533 n+3666\right) x-3\left(120 n^{2}+507 n+538\right)\right) y=0 .
\end{aligned}
$$

Figure 1: Differential equation for $y=H_{n}^{(3,2)}(x)$. 
determinant $H_{n}=\operatorname{det}\left[a_{i+j}(x)\right]_{0 \leq i, j \leq n}$ as

$$
d_{x} H_{n}=\operatorname{Tr}\left(A_{n}^{-1} d_{x} A_{n}\right) H_{n}
$$

where

$$
d_{x} A_{n}=d_{x} A_{n}(x)=\left[d_{x} a_{i+j}(x)\right]_{0 \leq i, j \leq n} .
$$

The obvious thing to try at this point is to look for expansions of the form

$$
d_{x} A_{n}=R A_{n}+A_{n} S
$$

so that we could prove that

$$
d_{x} \operatorname{det}\left(A_{n}\right)=\operatorname{Tr}(R+S) \operatorname{det}\left(A_{n}\right)
$$

This search also proved fruitless, and in retrospect Lemma 3 suggests that it will be very difficult to find $R$ and $S$ because

$$
(x-3)(2 x-3)(4 x-3) \operatorname{Tr}(R+S)=8 n x^{2}-6(5 n+1) x-3\left(9 n^{2}+13 n+8\right)+2(4 n+3) \frac{K_{n}}{H_{n}}
$$

and experiments suggest that $K_{n}$ and $H_{n}$ are often relatively prime.

So you have to feed some carefully selected facts into $d_{x} A_{n}$ to get a useful expansion of $d_{x} H_{n}$. Initially the first order differential equation

$$
x(x-1) d_{x} a_{n}(x)-(n(x-3)-2) a_{n}(x)-(3 n+2) a_{n}(0)=0
$$

looks like the fact needed. However this does not work because we never figured out what to do with the evaluation of the term

$$
\operatorname{Tr}\left(A_{n}^{-1}\left[(3 i+3 j+2) a_{i+j}(0)\right]_{0 \leq i, j \leq n}\right) .
$$

So at this point we started searching for matrices that behaved well after being evaluated by the operator

$$
\operatorname{Tr}\left(A_{n}^{-1} *\right)
$$

By "behaves well" we mean that the calculations return a single determinant, or at most a linear combination of a just a few determinants, thus avoiding the expansion of $H_{n}$ as a sum of a large number of determinants. We quickly learned about the first two columns in Table 1 in Section 5. But this was not sufficient because $d_{x} A_{n}$ cannot be expressed as a sum of the matrices defined in these two columns.

At this point we recalled some results in the literature $[5,6]$ that related derivatives to convolutions. We realized that what this work told us was that the operator in (166) works out very nicely on $\left[c_{i+j+1}\right],\left[c_{i+j}\right],\left[c_{i+j-1}\right]$, i.e. the trace came out to be a single determinant as already shown in Table 1. 
This gave us enough tools to find our first identity, given as (11) in Lemma 1. Using this identity we have enough to express the first and second derivatives of $H_{n}$ as linear combinations of small numbers of determinants. In particular in the examples of this paper, the first derivative of $H_{n}$ is expressed as a linear combination of $H_{n}$ and $K_{n}$. The second derivative is expressed as a linear combination of $H_{n}, K_{n}, M_{n}$ and $N_{n}$. This process can easily be continued to higher derivatives though the computations get messier at each derivative.

If the first identity (Lemma 1 ) is weakened to include terms like $a_{n+2}$ and $c_{n+2}$, the above process still works. The only difference is that the derivatives have more distinct determinant summands. The point of the above discussion is that we cannot handle any $(\beta, \alpha)$-case in which there does not exist a first identity of this kind. So far there are a dozen cases, some not of the $(\beta, \alpha)$ type, which have a first identity that we can handle, and we mention $(3,0),(3,2)$, and $(2,2)$ as examples of these.

The role of the second (Lemma 2) and third identities ( 102) is to prove linear relationships between the determinants that are generated by the above process of differentiating $H_{n}$.

Of these three identities, the proof is most sensitive to the form of the second identity. In the $(3,2)$-case, the second identity involves $a_{n+3}$ terms. While this version of the second identity can still be used to prove linear relationships between determinants, there are not enough linear relationships, and a proof of the fourth order differential equation for the $(3,2)$-case does not seem possible with the tools of this paper.

Another difficulty that needs to be overcome is the problem of finding and proving the third identity. Our original approach was to guess the form of the third identity and thus reduce the problem to binomial identity proving techniques. This works quite well for the $(2,1)$-case where the third identity has an explicit form:

$$
\begin{aligned}
\sum_{i=0}^{n+2}(-1)^{i}\left\{2\left(\begin{array}{c}
n+1+i \\
n+1-i
\end{array}\right)+\left(\begin{array}{c}
n+1+i \\
n+2-i
\end{array}\right)\right. & \\
& \left.+\left(2\left(\begin{array}{c}
n+1+i \\
n-i
\end{array}\right)+\left(\begin{array}{c}
n+1+i \\
n+1-i
\end{array}\right)\right) x\right\} a_{i+m}(x)=0
\end{aligned}
$$

for $0 \leq m \leq n$.

Though we were eventually able to guess the third identity in the $(3,1)$-case, its form was hardly enlightening. In addition, the identity was so complex that even the job of applying automated tools to prove it would be a major undertaking. So the whole process had arrived at an impasse.

The way forward was a sidestep via an existence theorem (Section 4). The third identity (once proved) said that the binomial identities we wished to prove actually existed, and were unique, and you only had to know a few of their components explicitly to show 
that the right hand side of (96) vanished. The third identity for the $(3,1)$-case is $(102)$. This identity is proved via the generating function of the $a_{n}(x)$ and from the explicit linear relationships governing the determinants $H_{n}(x), K_{n}(x), N_{n}(x)$ at special values of $x$.

Of special mention is the form of the generating function given in Lemma 5 . It is important to know that this is not the form that emerges in the straightforward derivation of the generating function, as was done in Lemma 9. We were surprised that we could not use this form directly to prove the third identity. It took a long time to discover the form in Lemma 5. This latter form worked, and this concluded the proof of the $(3,1)$-case.

To sum up, a lot of things have to fall right in place perfectly to evaluate a $H_{n}^{(\beta, \alpha)}(x)$.

\section{Additional results on Hankel determinants}

The proof technique presented here is applicable to Hankel determinants of polynomials other than the $a_{k}^{(\beta, \alpha)}$. Here we give the necessary ingredients, i.e. the three identities required, for the proof of the differential equation satisfied for a few of these but omit the proofs of the theorems and the construction of the explicit power series solutions.

As it happens, the product form evaluation (4) of the (3,0)-case at $x=0$ is not among the many binomial Hankel determinant evaluations that appear in Krattenthaler ([8], Theorem 31), and we start with the $(3,0)$-case as the first example.

\section{Example}

First identity for the $(3,0)$-case is:

$$
\begin{aligned}
& 3(x-3) x(4 x-3) d_{x} a_{n}-(4(2 x-3) n+2(2 x-5)) a_{n+1} \\
& +\left(27(2 x-3) n+3\left(4 x^{2}-3 x-9\right)\right) a_{n}-(x-1) c_{n+1}+27(x-1) c_{n}=0 .
\end{aligned}
$$

Second identity for the $(3,0)$-case:

$$
\begin{aligned}
& \left(4(2 x-3)^{2}(5 x-3) n+2(2 x-3)(5 x-3)(6 x-11)\right) a_{n+2} \\
& -\left(81\left(8 x^{3}-24 x^{2}+27 x-9\right) n+18\left(37 x^{3}-123 x^{2}+153 x-54\right)\right) a_{n+1} \\
& +\left(729 x^{3} n+486 x^{3}\right) a_{n}+4(x-1)(2 x-3)(5 x-3) c_{n+2} \\
& -3\left(40 x^{4}-30 x^{3}-207 x^{2}+270 x-81\right) c_{n+1}+162 x^{2}\left(5 x^{2}-15 x+9\right) c_{n}=0 .
\end{aligned}
$$

The generating function of the $a_{k}$ for the third identity for the $(3,0)$-case:

$$
f(x, y)=\frac{-(2 x-3) t-3 x}{\left(x^{2}(9 y-4)+10 x-6\right) t+(x-3)(4 x-3)}
$$

where $t^{3} y=t-1$ 
Theorem 6 The Hankel determinant for the (3,0)-case satisfies the differential equation

$$
\begin{gathered}
(x-3)(2 x-3)(5 x-3) d_{x}^{2} y-2\left(10(n-1) x^{2}-9(3 n-4) x-9(n+5)\right) d_{x} y \\
+n(10(n-1) x-3(n-7)) y=0 .
\end{gathered}
$$

In the $(3,0)$-case, in addition to the stated product form evaluation at $x=0$ given in (4), at $x=3$ and surprisingly also at $x=\frac{3}{2}$ the determinant is given by a simple product. We omit the proofs here but record these evaluations below. Details of this case will be presented in $[2]$.

$$
\begin{aligned}
H_{n}^{(3,0)}(3) & =\frac{(3 n) !(3 n+2) !}{2\left(n !^{2}\right)} \prod_{i=1}^{n} \frac{3(6 i-5) !(2 i) !(2 i-1)}{(4 i+1) !(4 i-1) !} \\
H_{n}^{(3,0)}\left(\frac{3}{2}\right) & =\prod_{i=1}^{n} \frac{27(6 i-5) !(3 i-1)(3 i-2)(2 i-1) !}{2(4 i-1) !(4 i-3)(4 i-4) !}
\end{aligned}
$$

\section{Example}

Next, take

$$
a_{k}(x)=\sum_{m=0}^{k}\left(\begin{array}{c}
3 k-2 m \\
k-m
\end{array}\right) x^{m} .
$$

The first identity for the polynomials in (170):

$$
\begin{aligned}
& x(2 x-9)(4 x+9) d_{x} a_{n}-(36 n+30) a_{n+1}+\left(243 n+8 x^{2}+18 x+81\right) a_{n} \\
& -12 c_{n+1}-\left(8 x^{2}-36 x-81\right) c_{n}+27 x(2 x-9) c_{n-1}=0 .
\end{aligned}
$$

The second identity for the polynomials in (170):

$$
\begin{aligned}
& (36(2 x+3) n+66(2 x+3)) a_{n+2} \\
& -\left(\left(32 x^{3}+486 x+729\right) n+12\left(4 x^{3}+4 x^{2}+54 x+81\right)\right) a_{n+1} \\
& +\left(216 x^{3} n+108 x^{2}(2 x+3)\right) a_{n}+12(2 x+3) c_{n+2} \\
& +\left(16 x^{3}-72 x^{2}-378 x-243\right) c_{n+1} \\
& +2 x\left(8 x^{3}-90 x^{2}+243 x+729\right) c_{n}-54 x^{3}(2 x-9) c_{n-1}=0 .
\end{aligned}
$$

The generating function of the $a_{k}$ for the third identity for the polynomials in (170):

$$
f(x, y)=\frac{3 t+2 x}{(x(4 x-9) y-6) t+9+2 x-6 x^{2} y}
$$

where $t^{3} y=t-1$.

Theorem 7 The Hankel determinant of the polynomials in (170) satisfies the differential equation

$$
(2 x+3)(2 x-9) d_{x}^{2} y+4(2(n+2) x-9(n+1)) d_{x} y-12 n(n+1) y=0 .
$$




\section{Example}

What we refer to as the "aex"-case (for exceptional) is the Hankel determinant where

$$
a_{k}(x)=\frac{1}{k+1} \sum_{m=0}^{k}\left(\begin{array}{c}
3 k-m \\
k-m
\end{array}\right)(m+1)(m+2) x^{m} .
$$

The first identity for the aex-case:

$$
\begin{aligned}
& x(x-3)(7 x-3) d_{x} a_{n}-(4(x-1) n+14(x-1)) a_{n+1} \\
& +(27(x-1) n+3(x-1)(11 x+3)) a_{n}-6(x-1)^{2} c_{n}+6 x^{3} c_{n-1}=0 .
\end{aligned}
$$

The second identity for the aex-case:

$$
\begin{aligned}
& \left(4(x-1)^{2}(3 x-1) n+18(x-1)^{2}(3 x-1)\right) a_{n+2} \\
& +\left(\left(-113 x^{3}+189 x^{2}-135 x+27\right) n-4\left(30 x^{4}-19 x^{3}-21 x^{2}+39 x-9\right)\right) a_{n+1} \\
& +\left(216 x^{3} n+12 x^{3}\left(7 x^{2}-2 x+15\right)\right) a_{n} \\
& +6(x-1)^{3}(3 x-1) c_{n+1}-6 x^{2}(x-1)\left(10 x^{2}-17 x+9\right) c_{n}+6 x^{5}(7 x-9) c_{n-1}=0 .
\end{aligned}
$$

The generating function of the $a_{k}$ for the third identity for the aex-case:

$$
f(x, y)=\frac{2(x-1)^{2} \tau-2(x-1)^{2}-2(3 x-1)}{\left(-2 y x^{2}(x-3) \tau+2 y x^{2}(x-3)+6 x^{2} y-3 x+1\right.}
$$

where

$$
\tau=\sum_{k \geq 0} \frac{(3 k) !}{(2 k) !(k+1) !} y^{k}
$$

Theorem 8 The Hankel determinant of the polynomials in (171) satisfies the differential equation

$$
(3 x-1)(x-1)(x-3) d_{x}^{2} y-2\left(3 n x^{2}-8 n x-3(n+4)\right) d_{x} y+3 n(n+1)(x-1) y=0 .
$$

In the aex-case, the determinant is given by a simple product for $x=\frac{3}{7}$ as shown below:

$$
H_{n}\left(\frac{3}{7}\right)=\prod_{i=0}^{n} \frac{2(6 i+7) !(2 i+1) !}{7(4 i+5) !(4 i+3) !} .
$$

\section{Example}

We can also evaluate the Hankel determinants as an almost product for

$$
a_{k}(x)=\sum_{m=0}^{k}\left(\begin{array}{c}
3 k+1 \\
k-m
\end{array}\right) x^{m}
$$


These polynomials are related to the polynomials $a_{k}^{(3,0)}(x)$ of Example 8 by the simple transformation

$$
\sum_{m=0}^{k}\left(\begin{array}{c}
3 k+1 \\
k-m
\end{array}\right) x^{m}=\sum_{m=0}^{k}\left(\begin{array}{c}
3 k-m \\
k-m
\end{array}\right)(x+1)^{m}=a_{k}^{(3,0)}(x+1)
$$

which is a special case of the transformation formula

$$
{ }_{2} F_{1}\left[\begin{array}{c}
a,-n \\
b
\end{array} ; z\right]=\frac{(c-a)_{n}}{(c)_{n}} F_{1}\left[\begin{array}{c}
-n, a \\
1+a-c-n
\end{array} ; 1-z\right]
$$

where $n$ is a nonnegative integer [13].

Therefore we have

Theorem 9 The Hankel determinant of the polynomials in (173) satisfies the differential equation

$$
\begin{aligned}
& (x-2)(2 x-1)(5 x+2) d_{x}^{2} y \\
& -2\left(10(n-1) x^{2}-(7 n-16) x-26 n-19\right) d_{x} y+n(10(n-1) x+7 n+11) y=0 .
\end{aligned}
$$

\section{Example}

Finally we have an alternate evaluation of $H_{n}^{(3,1)}(x)$ at $x=1$.

Theorem 10 Suppose $a_{k}(x)$ is defined as in (7) and $H_{n}(x)=\operatorname{det}\left[a_{i+j}(x)\right]_{0 \leq i, j \leq n}$. Then

$$
H_{n}(x)=\prod_{i=1}^{n} \frac{(6 i+4) !(2 i+1) !}{2(4 i+2) !(4 i+3) !} \sum_{i=0}^{n} \frac{(-1)^{i} n !(4 n+3) ! !(3 n+i+2) !(x-1)^{i}}{(3 n+2) ! i !(n-i) !(4 n+2 i+3) ! !}
$$

As in Corollary 4, taking $x=0$ and $x=1$ in (175) we obtain the following alternate evaluations.

\section{Corollary 9}

$$
\begin{aligned}
& \operatorname{det}\left[\left(\begin{array}{c}
3(i+j)+1 \\
i+j
\end{array}\right)\right]_{0 \leq i, j \leq n}= \\
& \prod_{i=1}^{n} \frac{(6 i+4) !(2 i+1) !}{2(4 i+2) !(4 i+3) !} \sum_{i=0}^{n} \frac{n !(4 n+3) ! !(3 n+i+2) !}{(3 n+2) ! i !(n-i) !(4 n+2 i+3) ! !}, \\
& \operatorname{det}\left[\left(\begin{array}{c}
3(i+j)+2 \\
i+j
\end{array}\right)\right]_{0 \leq i, j \leq n}=\prod_{i=1}^{n} \frac{(6 i+4) !(2 i+1) !}{2(4 i+2) !(4 i+3) !} .
\end{aligned}
$$

The first evaluation in Corollary 9 is the expression given in (6) for $H_{n}^{(3,1)}$. It is special as there are no cancellations on the right. The second evaluation is identical in form to the known product in (3) for $H_{n}^{(3,2)}$. 
There are other variants on the polynomials $a_{k}^{(\beta, \alpha)}$ which experiments suggest satisfy differential equations. A particularly unusual example are the Hankel determinants for

$$
a_{k}(x)=\sum_{m=0}^{k+1}\left(\begin{array}{c}
3 k+4-m \\
k+1-m
\end{array}\right) x^{m} .
$$

These determinants satisfy a third order differential equation, but the coefficients are very large and not round, making it hard to guess what they are.

Going back to the Hankel determinants $H_{n}^{(\beta, \alpha)}$, some of these are governed by a second order differential equation, such as $(3,0),(3,1)$, and $(2,1)$. There are also non- $(\beta, \alpha)$-cases governed by a second order differential equation such as the aex-case in Example 8 .

In forthcoming work, we plan to refine the methods of this paper so that the approach sidesteps the complications arising from the nonlinear terms that are produced by the trace calculations of the derivatives. There is also ongoing work on Hankel determinants related to other interesting families of polynomials. In the family $(2, r)$, for example, the three cases $r=0,1,2$ have product evaluations, but for $r \geq 3$, the evaluations are no longer products. We also encounter new phenomena in these cases, such as higher order differential equations and case-splitting into residue classes.

We remark that experimentally we know that both $(2,3)$ and $(3,2)$-cases satisfy fourth order differential equations. For the $(2,3)$-case, the polynomial in front of the fourth derivative has degree 11.

Acknowledgments: We would like to thank Julius Borcea, Ira Gessel, Christian Krattenthaler, Boris Shapiro, and Doron Zeilberger. We would also like to thank the referee for a careful reading of this paper and for the comments on known results regarding hypergeometric transformations, which we have incorporated. The suggestions considerably improved the presentation. 


\section{Appendix I: The generating function of the $a_{k}^{(\beta, \alpha)}(x)$}

In Lemma 9, we give a closed form of the generating function $f(x, y)$ defined in $(104)$.

Lemma 9 Suppose $a_{k}(x)$ is as defined in (10) and $f$ is as in (104). Then

$$
f(x, y)=\frac{t^{\alpha+1}}{(\beta+(1-\beta) t)\left(1-x y t^{\beta-1}\right)}
$$

where

$$
t^{\beta} y=t-1
$$

Proof Changing the order of summation and rearranging

$$
f(x, y)=\sum_{m \geq 0} x^{m} y^{m} \sum_{n \geq 0}\left(\begin{array}{c}
\beta n+(\beta-1) m+\alpha \\
n
\end{array}\right) y^{n} .
$$

It is known [11] that

$$
\sum_{n \geq 0}\left(\begin{array}{c}
\alpha+\beta n \\
n
\end{array}\right) y^{n}=\frac{t^{\alpha+1}}{\beta+(1-\beta) t}
$$

where $t$ satisfies (177). For our generating function, $\beta$ is the same but $\alpha$ in (178) is replaced by $(\beta-1) m+\alpha$. Using these parameters we obtain

$$
f(x, y)=\sum_{m \geq 0} x^{m} y^{m} \frac{t^{(\beta-1) m+\alpha+1}}{\beta+(1-\beta) t}=\frac{t^{\alpha+1}}{(\beta+(1-\beta) t)\left(1-x y t^{\beta-1}\right)}
$$

where $t$ satisfies (177).

Note that using the Lagrange inversion formula, $t$ can be expanded as

$$
t=\sum_{k \geq 0} \frac{(\beta k) !}{((\beta-1) k+1) ! k !} y^{k}=1+y+\beta y^{2}+\frac{\beta(3 \beta-1)}{2} y^{3}+\cdots
$$

\section{Proof of Lemma 5:}

Proof The expression (179) for $f$ with $\beta=3$ and $\alpha=1$ can be rewritten in the form (105) since

$$
\frac{t^{2}}{(3-2 t)\left(1-x y t^{2}\right)}-\frac{4 x+2 t-6}{(x-3)(4 x-3) y(2 t-3)-(x-1)(27 y-4)}
$$

is equal to

$$
\frac{\left(t-1-t^{3} y\right)(2 t x+6 x-9)}{(3-2 t)\left(1-x y t^{2}\right)\left(4 t x^{2} y-6 x^{2} y-15 t x y+9 x y+9 t y+2 x-2\right)}
$$

and the numerator has $t-1-t^{3} y$ as factor, but this is zero by (177). This gives the form of the generating function $f$ for the $(3,1)$-case that is claimed in (105). 


\section{Proof of Lemma 8:}

Proof The proof is similar to the proof of Lemma 5 once we observe that the expression for the generating function

$$
f(x, y)=\frac{t^{2}}{(2-t)(1-x y t)}
$$

from Lemma 9 can be written as (144) since

$$
\frac{t^{2}}{(2-t)(1-x y t)}-\frac{t}{(x-2) y t+1-2 x y}=\frac{2 t\left(1-t+t^{2} y\right)}{(t-2)(1-x y t)(1-2 y t-2 x y-x y t)}
$$

and the right hand side vanishes since in this case $\beta=2$ and

$$
1-t+t^{2} y=0
$$

by Lemma 9 .

\section{Appendix II: Pairs of identities for the $(3,1)$ and $(2,1)$-cases.}

Proofs of the first two identities in the $(3,1)$-case are as follows:

Proof of Lemma 1:

Proof We make use of the generating function $f=f(x, y)$ of the $a_{k}$ 's in the form given by (179) in Appendix I. Passing to the generating functions, (11) is equivalent to the functional identity

$$
\begin{aligned}
(x-3)(2 x-3)( & 4 x-3) d_{x} f-4 y d_{y} \frac{f-1}{y}-6 \frac{f-1}{y}+\left(8 x^{2}-18 x+36\right) f \\
+ & 27 y d_{y} f-4\left(2 x^{2}-6 x+3\right) f^{2}+27\left(2 x^{2}-6 x+3\right) y f^{2}=0 .
\end{aligned}
$$

Using identity (177),

$$
d_{y} t=\frac{t^{3}}{1-3 y t^{2}} .
$$

Substituting this expression in the computation of $d_{y} f$, the left hand side of (183) can be simplified as

$$
\begin{aligned}
& \frac{2\left(t^{3} y-t+1\right)}{\left(1-3 y t^{2}\right)}\left(2 x t^{2}+12 x^{2} y t^{2}-90 x y t^{2}+54 y t^{2}+2 t^{2}+8 x^{2} t\right. \\
& \left.+x t-36 x^{2} y t+135 x y t-81 y t-15 t-8 x^{2}-3 x+9\right)
\end{aligned}
$$

which vanishes since $t^{3} y-t+1=0$ by (177). 


\section{Proof of Lemma 2:}

Proof We again use the generating function $f=f(x, y)$ of the $a_{k}$ 's in the form given by (179). The identity (13) is equivalent to

$$
\begin{aligned}
& 8(x-1) y d_{y} \frac{f-1-(4+x) y}{y^{2}}+20(x-1) \frac{f-1-(4+x) y}{y^{2}} \\
& -2\left(-81+135 x-72 x^{2}+16 x^{3}\right) y d_{y} \frac{f-1}{y}-2\left(-117+180 x-92 x^{2}+24 x^{3}\right) \frac{f-1}{y} \\
& +27(2 x-3)^{3} y d_{y} f+54(2 x-3)\left(3-4 x+2 x^{2}\right) f \\
& \left.+8(x-1)\left(3-6 x+2 x^{2}\right)\right) \frac{f^{2}-1}{y} \\
& +2\left(81-297 x+324 x^{2}-114 x^{3}+8 x^{4}\right) f^{2}-27 x(2 x-3)\left(9-12 x+2 x^{2}\right) y f^{2}=0 .
\end{aligned}
$$

Using the expression in (184) for $d_{y} t$ in the calculation of $d_{y} f$, the left hand side of (185) can be simplified as

$$
\begin{aligned}
& \frac{2\left(t^{3} y-t+1\right)}{(3-2 t)^{2} y^{2}\left(1-3 y t^{2}\right)\left(1-x y t^{2}\right)^{2}}\left(-72 t^{2} y^{2} x^{4}+16 t^{2} y x^{4}+56 t y x^{4}-72 y x^{4}\right. \\
& -108 t^{2} y^{2} x^{3}-16 t x^{3}-48 t^{2} y x^{3}-156 t y x^{3}+252 y x^{3}+16 x^{3}-4 t^{2} x^{2}+1134 t^{2} y^{2} x^{2} \\
& +14 t x^{2}+72 t^{2} y x^{2}+252 t y x^{2}-486 y x^{2}-10 x^{2}-1701 t^{2} y^{2} x+32 t x+18 t^{2} y x \\
& \left.-405 t y x+567 y x-24 x+4 t^{2}+729 t^{2} y^{2}-30 t-54 t^{2} y+243 t y-243 y+18\right)
\end{aligned}
$$

which vanishes since $t^{3} y-t+1=0$ by (177).

Proofs of the first two identities in the $(2,1)$-case are as follows:

\section{Proof of Lemma 6:}

Proof Passing to the generating functions in (131), we need to prove the identity

$$
2 x(x-2) d_{x} f-d_{y} f+4 y d_{y} f+2(x+1) f-(x-1) f^{2}+4(x-1) y f^{2}=0 .
$$

Using the expression

$$
d_{y} t=\frac{t^{2}}{1-2 y t}
$$

in the calculation of $d_{y} f$, the left hand side of the identity we want to prove can be simplified as

$$
\frac{2 t^{2}\left(t^{2} y-t+1\right)(2-t+2 x+4 t y-6 t x y)}{(2-t)^{2}(1-2 t y)(1-t x y)^{2}}
$$

which vanishes by (182).

The proof of the second identity for the $(2,1)$-case is as follows:

\section{Proof of Lemma 7:}


Proof Again passing to the generating functions in (132), we need to prove the identity

$$
\begin{aligned}
& x y d_{y} \frac{f-1-(x+3) y}{y^{2}}+2(x+1) \frac{f-1-(x+3) y}{y^{2}} \\
& -2 x(x+2) y d_{y} \frac{f-1}{y}-2\left(2 x^{2}+3 x+4\right) \frac{f-1}{y} \\
& +8 x^{2} y d_{y} f+12 x^{2} f+(x-1)(x-2) \frac{f^{2}-1}{y}-4(x-1)(x-2) f^{2}=0 .
\end{aligned}
$$

Using the expression for $f$ and the expression for $d_{y} t$ in (186), the left hand side of this expression can be simplified to

$$
\begin{gathered}
\frac{2\left(t^{2} y-t+1\right)}{(2-t)^{2} y^{2}(1-2 t y)(1-t x y)^{2}}\left(t^{2}-4+8 t y-4 t^{2} y-2 t^{3} y+8 t x y-2 t^{2} x y+t^{3} x y\right. \\
\left.-2 t^{2} x^{2} y+8 t^{3} y^{2}-16 t^{2} x y^{2}-4 t^{3} x y^{2}+8 t^{2} x^{2} y^{2}+4 t^{3} x^{2} y^{2}-4 t^{3} x^{3} y^{3}\right)
\end{gathered}
$$

which again vanishes by (182).

\section{Appendix III: On the degree of a class of Hankel Determinants}

Theorem 11 Let $p_{0}, p_{1}, \ldots, p_{n}$ and $q_{0}, q_{1}, \ldots, q_{n}$ be integer sequences. Further let $\gamma$ be real and $\alpha_{0}, \alpha_{1}, \ldots, \alpha_{n}$ and $\beta_{0}, \beta_{1}, \ldots, \beta_{n}$ be sequences of real numbers. Then the determinant

$$
\operatorname{det}\left[\sum_{0 \leq m \leq p_{i}+q_{j}}\left(\begin{array}{c}
\alpha_{i}+\beta_{j}+\gamma m \\
p_{i}+q_{j}-m
\end{array}\right) x^{m}\right]_{0 \leq i, j \leq n}
$$

as a polynomial in $x$ has degree $\leq \max \left\{\max p_{i}+\max q_{j}-n, 0\right\}$.

Proof We note the convention that the empty sum is zero, and the binomial coefficient in (187) is interpreted via the gamma function. The proof of the theorem is by induction. At each stage of the induction we make use of the following lemma.

Lemma 10 Let $p_{0}, p_{1}, \ldots, p_{n}$ and $q_{0}, q_{1}, \ldots, q_{n}$ be two integer sequences and let $a_{0}, a_{1}, \ldots$ be an infinite sequence of real numbers. Then the determinant

$$
\operatorname{det}\left[\sum_{0 \leq m \leq p_{i}+q_{j}} a_{p_{i}+q_{j}-m} x^{m}\right]_{0 \leq i, j \leq n}
$$

as a polynomial in $x$ has degree $\leq \max \left\{\max p_{i}+\max q_{j}-n, 0\right\}$. 
Proof By rearranging the indices, we can assume that the sequences $p_{0}, p_{1}, \ldots, p_{n}$ and $q_{0}, q_{1}, \ldots, q_{n}$ are nondecreasing without changing the conclusion of the lemma. Then

$$
\operatorname{det}\left[\sum_{0 \leq m \leq p_{i}+q_{j}} a_{p_{i}+q_{j}-m} x^{m}\right]_{0 \leq i, j \leq n}=y^{-\sum p_{i}-\sum q_{j}} \cdot \operatorname{det}\left[\sum_{0 \leq m \leq p_{i}+q_{j}} a_{m} y^{m}\right]_{0 \leq i, j \leq n}
$$

where $x y=1$. Therefore the analysis can focus on the degree of

$$
\operatorname{det}\left[\sum_{0 \leq m \leq p_{i}+q_{j}} a_{m} y^{m}\right]_{0 \leq i, j \leq n}
$$

as a polynomial in $y$. By elementary row operations, we see that this is equal to the following determinant

$$
\operatorname{det}\left[\left\{\begin{array}{cc}
\sum_{0 \leq m \leq p_{0}+q_{j}} a_{m} y^{m} & \text { if } i=0 \\
\sum_{0 \leq m ; p_{i-1}+q_{j}+1 \leq m \leq p_{i}+q_{j}} a_{m} y^{m} & \text { otherwise }
\end{array}\right]_{0 \leq i, j \leq n} .\right.
$$

Whenever $p_{n}+q_{n} \geq n$, the highest power of $y$ in this determinant is no more than

$$
\max \left\{\sum p_{i}+\sum q_{j}, 0\right\}
$$

and the lowest power of $y$ in this determinant is at least

$$
\max \left\{n+\sum_{i=0}^{n-1} p_{i}+\sum_{j=0}^{n-1} q_{j}, 0\right\}
$$

Now if we multiply this polynomial by $y^{-\sum p_{i}-\sum q_{j}}$ obtaining

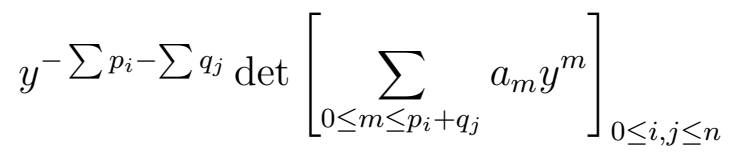

we get a polynomial in $y^{-1}$. The degree of this polynomial is no more than

$$
\max \left\{p_{n}+q_{n}-n, 0\right\} \text {. }
$$

Converting back to $x$ and remembering that $p_{i}$ and $q_{j}$ are nondecreasing gives the desired result.

Now we are ready to start the proof of Theorem 11. Let

$$
\begin{aligned}
\alpha_{i}^{\prime} & =\alpha_{i}+\gamma p_{i} \\
\beta_{j}^{\prime} & =\beta_{j}+\gamma q_{j} .
\end{aligned}
$$


With this change of variable,

$$
\left(\begin{array}{c}
\alpha_{i}+\beta_{j}+\gamma m \\
p_{i}+q_{j}-m
\end{array}\right)=\left(\begin{array}{c}
\alpha_{i}^{\prime}+\beta_{j}^{\prime}-\gamma\left(p_{i}+q_{j}-m\right) \\
p_{i}+q_{j}-m
\end{array}\right) .
$$

The following identity holds:

$$
\left(\begin{array}{c}
\alpha_{i}+\beta_{j}+\gamma m \\
p_{i}+q_{j}-m
\end{array}\right)=\sum_{r \geq 0} \sum_{s=0}^{p_{i}+q_{j}-m-r}\left(\begin{array}{c}
\alpha_{i}^{\prime} \\
r
\end{array}\right)\left(\begin{array}{c}
\beta_{j}^{\prime} \\
s
\end{array}\right)\left(\begin{array}{c}
-\gamma\left(p_{i}+q_{j}-m\right) \\
p_{i}-r+q_{j}-s-m
\end{array}\right) .
$$

This allows us to express the determinant as

$$
\operatorname{det}\left[\sum_{r \geq 0} \sum_{s \geq 0}\left(\begin{array}{c}
\alpha_{i}^{\prime} \\
r
\end{array}\right)\left(\begin{array}{c}
\beta_{j}^{\prime} \\
s
\end{array}\right)_{0 \leq m \leq p_{i}-r+q_{j}-s}\left(\begin{array}{c}
-\gamma\left(p_{i}+q_{j}-m\right) \\
p_{i}-r+q_{j}-s-m
\end{array}\right) x^{m}\right]_{0 \leq i, j \leq n}
$$

This determinant has an expansion as a sum of terms of the form

$$
\left(\prod_{i}\left(\begin{array}{c}
\alpha_{i}^{\prime} \\
r_{i}
\end{array}\right)\right)\left(\prod_{j}\left(\begin{array}{c}
\beta_{j}^{\prime} \\
s_{j}
\end{array}\right)\right) \cdot \operatorname{det}\left[\sum_{0 \leq m \leq p_{i}-r_{i}+q_{j}-s_{j}}\left(\begin{array}{c}
-\gamma p_{i}+-\gamma q_{j}+\gamma m \\
p_{i}-r_{i}+q_{j}-s_{j}-m
\end{array}\right) x^{m}\right]_{0 \leq i, j \leq n}
$$

over collections of nonnegative integers $r_{0}, r_{1}, \ldots, r_{n}$ and $s_{0}, s_{1}, \ldots, s_{n}$.

If all the $r_{i}$ and $s_{j}$ in any such collection are zero, then the corresponding determinant is as described in Lemma 10 and therefore has the appropriate degree as a polynomial in $x$. If any of the $r_{i}$ or $s_{j}$ are nonzero, then the determinant has the same form as that of Theorem 11 except that $p_{i}$ has been replaced with $p_{i}^{\prime}=p_{i}-r_{i}$ and $q_{j}^{\prime}=q_{j}-s_{j}$. This is the induction process.

Note that the induction process terminates, since eventually

$$
\max \left\{\max p_{i}^{\prime}+\max q_{j}^{\prime}-n, 0\right\}=0
$$

and in fact the resulting determinants in (187) become zero.

Corollary 10 The $(n+1) \times(n+1)$ Hankel determinant $H_{n}^{(\beta, \alpha)}$ defined by the sequence of polynomials in (2) is of degree at most $n$.

Proof $H_{n}^{(\beta, \alpha)}$ is of the type described in Theorem 11 where $p_{i}=q_{i}=i, \alpha_{i}=\beta i+\alpha$, $\beta_{i}=\beta i$, and $\gamma=-1$.

\section{References}

[1] Ö. Eğecioğlu, T. Redmond and C. Ryavec. From a Polynomial Riemann Hypothesis to Alternating Sign Matrices. The Electronic Journal of Combinatorics, Volume 8 (1), (2001), \#R36. 
[2] Ö. Ĕ̆gecioğlu, T. Redmond and C. Ryavec. Evaluation of a Special Hankel Determinant of Binomial Coefficients, in preparation.

[3] A. Lupas. A Characterization of the Appell Polynomials, Studia Univ. Babes-Bolyai, XXXIII, Nr. 2 , (1988) pp. 40-44.

[4] I. Gessel and G. Xin. The generating function of ternary trees and continued fractions, Electronic J. Combin. 13, (2006), no. 1, R53.

[5] K. Iwasaki, K. Kajiwara and T. Nakamura. Generating Function Associated with the Rational Solutions of the Painlevé II Equation, Journal of Physics A: Mathematical and General, Vol. 35, (2002), L207-L211.

[6] K. Kajiwara, T. Masuda, M. Noumi, Y. Ohta and Y. Yamada. Determinant Formulas for the Toda and Discrete Toda Equations, Funkcialaj Ekvacioj, Vol. 44, (2001), pp. 291-307.

[7] C. Krattenthaler, Advanced determinant calculus, Seminaire Lotharingien Combin. 42 ("The Andrews Festschrift") (1999), Article B42q, 67 pp.

[8] C. Krattenthaler, Advanced determinant calculus: a complement, Linear Algebra and its Appl. 411 (2005), pp. 68-166.

[9] D. E. Knuth. Overlapping Pfaffians, Electr. J. Comb. 3(2), (1996), R5 (13 pages).

[10] Liu, Lily L. and Y. Wang. A unified approach to polynomial sequences with only real zeros, 2006, eprint arXiv:math/0509207, Advances in Applied Mathematics (to appear).

[11] G. Pólya and G. Szegö. Problems and Theorems in Analysis, Vol. I, translated by D. Aeepli, Springer-Verlag New York 1972, Part 3, Problem 216, pp. 146, 349.

[12] C. Radoux. Addition formulas for polynomials built on classical combinatorial sequences, J. Comput. Appl. Math. 115, (2000), pp. 471-477.

[13] L. J. Slater. Generalized hypergeometric functions. Cambridge University Press, 1966, identity (1.8.10), pp. 34.

[14] J. Wimp. Hankel determinants of some polynomials arising in combinatorial analysis, Numerical Algorithms, Volume 24, Numbers 1-2, (2000), pp. 179-193 